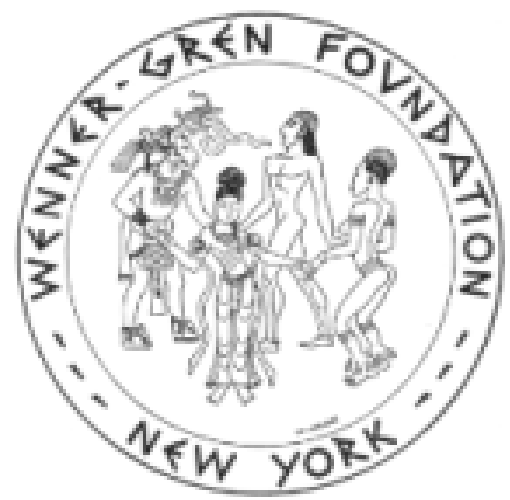

The Evolution of Tag-Based Cooperation in Humans: The Case for Accent Author(s): Emma Cohen

Reviewed work(s):

Source: Current Anthropology, Vol. 53, No. 5 (October 2012), pp. 588-616

Published by: The University of Chicago Press on behalf of Wenner-Gren Foundation for Anthropological

Research

Stable URL: http://www.jstor.org/stable/10.1086/667654

Accessed: $21 / 11 / 2012$ 06:56

Your use of the JSTOR archive indicates your acceptance of the Terms \& Conditions of Use, available at http://www.jstor.org/page/info/about/policies/terms.jsp

JSTOR is a not-for-profit service that helps scholars, researchers, and students discover, use, and build upon a wide range of content in a trusted digital archive. We use information technology and tools to increase productivity and facilitate new forms of scholarship. For more information about JSTOR, please contact support@ jstor.org.

The University of Chicago Press and Wenner-Gren Foundation for Anthropological Research are collaborating with JSTOR to digitize, preserve and extend access to Current Anthropology. 


\title{
The Evolution of Tag-Based Cooperation in Humans
}

\author{
The Case for Accent
}

\author{
by Emma Cohen
}

\begin{abstract}
Recent game-theoretic simulation and analytical models have demonstrated that cooperative strategies mediated by indicators of cooperative potential, or "tags," can invade, spread, and resist invasion by noncooperators across a range of population-structure and cost-benefit scenarios. The plausibility of these models is potentially relevant for human evolutionary accounts insofar as humans possess some phenotypic trait that could serve as a reliable tag. Linguistic markers, such as accent and dialect, have frequently been either cursorily defended or promptly dismissed as satisfying the criteria of a reliable and evolutionarily viable tag. This paper integrates evidence from a range of disciplines to develop and assess the claim that speech accent mediated the evolution of tag-based cooperation in humans. Existing evidence warrants the preliminary conclusion that accent markers meet the demands of an evolutionarily viable tag and potentially afforded a cost-effective solution to the challenges of maintaining viable cooperative relationships in diffuse, regional social networks.
\end{abstract}

\section{Introduction}

The scientific study of large-scale human sociality is located at the intersection of a range of disciplines, from population biology to sociocultural anthropology (e.g., Foley and Gamble 2009; Gardner and Grafen 2009; Johnson and Earle 2000; West, Griffin, and Gardner 2006b). Identifying the mechanisms regulating the costs and benefits of cooperation among individuals in structured populations is a central and recurrent problem across this broad disciplinary spectrum.

The problem of cooperation is ultimately a problem of assortment among individuals_cooperators (i.e., individuals who bestow benefits on others, potentially at a cost to self) must assort with the cooperation, not defection, of others if they are to reap benefits from their behavior (Eshel and Cavalli-Sforza 1982; Fletcher and Doebeli 2009; Hamilton 1975; Nowak, Tarnita, and Antal 2010). In recent years, considerable scholarly attention has focused on identifying mechanisms of assortment, producing increasingly sophisticated models of the evolution and maintenance of cooperation in terms of genetic, populational, environmental, and cultural mechanisms (see, e.g., Lehmann and Keller 2006 target article and commentaries).

Emma Cohen is a University Lecturer in the Institute of Cognitive and Evolutionary Anthropology of the University of Oxford (64 Banbury Road, Oxford OX2 6PN, United Kingdom [emma .cohen@anthro.ox.ac.uk]). This paper was submitted 4 XII 10 and accepted 1 I 12.
Some recent approaches have breathed new life into an old idea, demonstrating via computer simulations that cooperative strategies based on discernible, phenotypic tags (e.g., cooperate only with those who share one's phenotypic trait "X") can emerge and be sustained under a wide variety of conditions (e.g., Antal et al. 2009; Axelrod, Hammond, and Grafen 2004; Hammond and Axelrod 2006a, 2006b; Ihara 2011; Riolo, Cohen, and Axelrod 2001; Shultz, Hartshorn, and Hammond 2008; Shultz, Hartshorn, and Kaznatcheev 2009; Spector and Klein 2006; Traulsen and Schuster 2003). This paper considers the relevance of these developments for our understanding of the evolution of human cooperation.

The logical possibility of tag-based strategies is only potentially interesting and relevant for an account of human cooperation to the extent that humans possess some phenotypic trait that could plausibly serve as a reliable tag. The evolutionary viability of tag-based cooperation-whether via genetic or cultural traits-has generally been promptly rejected in the case of humans. Cooperative strategies based on genetic tags, such as "greenbeards," require a tight genetic link between the phenotypic tag (the greenbeard) and the behavior (contingent cooperation). They are therefore highly vulnerable to exploitation and invasion by mutant defectorsegoists who display the right tag but who do not cooperate (Dawkins 1976; Gardner and West 2010; Hamilton 1964). Although now documented in some cases in the biological world, it is generally agreed that the complexity of the greenbeard mechanism and its vulnerability to "falsebeard" invasion render such a genetically based guide to assortment extremely rare in nature (Gardner and West 2010). Alternative 
mechanisms guiding the assortment of genealogical kin have received considerably more attention in the human cooperation literature. Such "armpit mechanisms" (Dawkins 1982), sustained through detection mechanisms or population viscosity, can help explain the evolution of cooperation among kin but are less relevant to explaining contingent cooperation among unrelated or distantly related individuals in high-dispersal scenarios.

In contrast to greenbeards, symbolic tags are abundant in culture in the form of flags, badges, cuisines, tattoos, and hairstyles, and these often serve to channel cooperation. It is generally assumed, however, that they, like the roguish greenbeard, are so vulnerable to imitation that none could plausibly form the evolutionary foundation of any fitness-bestowing tag-based cooperation strategy among strangers (Boyd and Richerson 2005; McElreath, Boyd, and Richerson 2003; Richerson and Boyd 2005). Copycat defectors, who bear the tag but not the behavioral strategy (i.e., are noncooperators), can easily take advantage of a conditional cooperator's unwitting bestowal of benefits, while avoiding any costs, thereby fracturing the assortment mechanism required for cooperation to evolve. The relative fitness of the cooperator is reduced and cooperation is selected against. Increasing the costs of copying the tag can potentially attenuate this threat, but such "costly signaling" is generally considered important only after the cooperative social group and the evolved social-cognitive mechanisms permitting it have already formed (e.g., as a boundary policing mechanism, or commitment signal; Sosis, Kress, and Boster 2007). By such accounts, group-level cultural institutions ultimately explain the emergence of cooperation and in-group preferences within large communities of unrelated individuals (e.g., Boyd and Richerson 1985; Richerson and Boyd 2005). Tag-based cooperation in humans is therefore rejected as a plausible explanation for the emergence of the large-scale cooperation among strangers that characterizes modern human sociality (Henrich 2004).

Nevertheless, some theorists disagree. They argue that not all cultural tags are created equally. Whereas school ties, club memberships, tribal customs, and religious creeds are potentially vulnerable to exploitation, some markers, such as language and accent, are hard to fake and hard to hide: "hiding one's native accent in a foreign language is nearly impossible" (Sigmund and Nowak 2001:403; see also Irwin 1987; Kinzler, Dupoux, and Spelke 2007; Kinzler et al. 2009; Nettle 1999a; Nettle and Dunbar 1997; Roberts 2008; Tooby and Cosmides 1989; Traulsen 2008). Furthermore, although faking a nonnative accent poses considerable challenges in adulthood, the child acquires a native accent for free via early developing vocal imitation and language acquisition mechanisms. The reliability and cost efficiency of speech accent therefore appear to permit, or at least do not preclude, an evolutionary account of human cooperation that is based on a culturally acquired tag.

The suggestion that linguistic cues to social identity may have been important in human social evolution is not new (e.g., Baker 2002; Nettle and Dunbar 1997). But claims have remained unelaborated and underexplored in light of relevant theoretical and empirical developments across a range of disciplines. The minimal criteria that linguistic tags need to fulfill in order to sustain the assortment of cooperators have not been systematically identified nor evaluated against the relevant evidence. The purpose of this paper is to consider the plausibility of tag-based cooperation via linguistic cues as (part of) an explanation for the emergence and maintenance of human cooperation beyond the local group. Integrating disparate findings in anthropology, biology, linguistics, archaeology, and psychology, I assess the case for an integral role of linguistic assortative markers in the evolution of cooperation in sizable human groups.

The paper is divided into four sections. The first section reviews relevant literature on tag-based cooperation and outlines the requisite criteria of a reliable tag. The second section assesses whether linguistic cues, and particularly accent, meet these criteria and therefore may have served as an evolutionarily viable tag for the orientation of discriminatory cooperative strategies. The burden of theoretical plausibility, and the weight of current debate, rests largely on the issue of tag reliability. Accordingly, disproportionate space is dedicated to this section and to the discussion of empirical literature of relevance. The third section considers the historical conditions that may have permitted the emergence of language-based discrimination strategies in human social evolution. By way of conclusion, the final section summarizes a series of empirical questions and hypotheses arising from an accent-tag account.

\section{Tag-Based Cooperation}

Cooperation is here defined as any behavior that provides a fitness benefit to another individual (i.e., that benefits the genetic contribution that the individual makes to future generations; Grafen 2006); any such behavior that incurs a cost to the cooperator is altruistic cooperation, or altruism (see West, Griffin, and Gardner 2006b for further discussion of these and related terms). The fundamental condition necessary for the evolution and maintenance of cooperation in any population is that the costs of cooperation are offset by the benefits received from others, such that cooperators reap a net fitness advantage (relative to noncooperators). More precisely, there must be assortment between carriers of the cooperative strategy (whether genetically or culturally inherited) and the benefits they receive via the cooperative behaviors of others (Fletcher and Doebeli 2009). This is explained by the following basic principles of selection: any trait (cooperative or not) that causes its carrier to end up worse off than those individuals with whom the carrier interacts can only evolve if the benefits received from others make up for this disadvantage; for the trait to increase in frequency, its carriers must, on average, accrue more direct fitness benefits than average population members. On this view, identifying the 
mechanisms that enable cooperators to assort with other cooperators and not with defectors (individuals whose behaviors ultimately incur a fitness cost to the cooperator) is "the biologically most relevant problem for understanding cooperation" (Fletcher and Doebeli 2009:18; see also Nowak, Tarnita, and Antal 2010).

Many solutions to this problem have been advanced in recent decades. Since Hamilton's groundbreaking work (1964), in which he formalized Fisher's (1930) initial observation that genes can spread not only through their own transmission but through transmission of copies in other individuals, theoretical and empirical work has focused on identifying mechanisms of assortment that impact an individual's reproductive success (direct fitness) as well as that of his genetic kin (most commonly via shared descent; indirect fitness). Assortment mechanisms now standard, though still much debated, in the literature include kin selection, reciprocity, and multilevel selection (variously underpinned by mechanisms of population viscosity via limited dispersal of offspring, kin recognition, conditional cooperation or tit-fortat reciprocity, partner choice and partner switching, punishment, reward, policing, reputation, and competition among groups; see West, Griffin, and Gardner 2006b; West et al. 2006a).

In modern humans, adaptations for cooperation permit a variety of fitness-enhancing activities, such as cooperative hunting and foraging, defense against predators, and access to mates, while avoiding the fatal consequences of free-riding (e.g., Hill 2002; Sober and Wilson 1998). The history of these adaptations, and their human uniqueness, is a core focus in the study of human evolution and in comparative research on our closest evolutionary relatives-chimpanzees, bonobos, gorillas, and orangutans.

Some theorists have proposed that the unique ways in which humans organize social life-and, in particular, the ways they solve cooperation problems in large, complex societies - challenge existing biological theory and strongly suggest additional evolutionary adaptations to those previously proposed. For instance, "strong reciprocity" (the propensity to help others, including strangers in anonymous encounters, and to punish those who do not help others) has been presented as a novel evolutionary mechanism that explains cooperation among nonrelatives in the absence of reputational consequences and/or opportunities for repeated interaction (e.g., Fehr and Fischbacher 2003; Gintis 2000; Gintis et al. 2003). The benefit of this propensity is meted out at the group level. Where groups are sufficiently large as to render average genetic relatedness extremely low, it is argued that cooperation in such interactions cannot be explained in terms of inclusive fitness benefits (Bowles and Gintis 2004:18).

Many biologists and others have challenged the assertion that standard biological theory is insufficient to explain strong reciprocity (e.g., Burnham and Johnson 2005; Lehmann et al. 2007; West, Griffin, and Gardner 2006b; West, El Mouden, and Gardner 2010). Rather, purportedly anomalous behaviors are neither unique to humans nor inexplicable in terms of established mechanisms in biological theory, and proposed "new" mechanisms are often just reformulations of phenomena already well established and mathematically formalized in the biological literature. Lehmann et al. (2007) have shown, for example, that the evolution of strong reciprocity relies upon standard benefits to self and kin. Their models, which take explicit account of the consequences of dispersal and migration for intragroup genetic relatedness, demonstrate that altruism provides both direct and indirect fitness benefits.

Recently, several models in theoretical biology have investigated the logical plausibility of assortment among strangers based on observable, arbitrary traits—or "tags" — that signal cooperative potential. In these models, cooperators exploiting a tag-based strategy discriminate among potential cooperative partners according to the phenotypic tags they display. Cooperative behavior is contingent on one's interaction partner bearing the "right tag" (determined according to how the strategy is configured). Related models premised on similarity recognition (e.g., to self) have also been developed (e.g., Antal et al. 2009; Traulsen 2008).

Axelrod, Hammond, and Grafen (2004) specify three coevolutionary parameters governing the evolution of cooperation via tag-based assortative strategies: (1) the discriminatory strategies governing behavior; (2) the reliability of the tags on which the behavior may be conditioned; and (3) the population structure that determines who interacts with whom.

Using agent-based simulation techniques, they investigated the relative success of various tag-mediated strategies, such as cooperate or defect with those who share one's tag, and cooperate or defect with those who do not share one's tag (yielding four possible strategies). With a population structure that maintains offspring close to parents and a sufficiently high benefit-to-cost ratio, contingent cooperation stabilized within $77 \%$ of the population. Critically, because tags and their cooperative strategies are not linked (as they are, e.g., in a greenbeard strategy), the model explicitly allowed for the possibility of egoists who share the same tag as cooperators but who free-ride on the cooperation of fellow tag bearers. The success of cooperators in the face of such threats is explained in terms of the spatial dynamics of the model at the level of the region. Interactions are local (in other words, occurring among neighbors), and dispersal of offspring is limited. This allows successful agents and their offspring to rapidly form coherent regions. Whereas egoists receive no help from those in their region, discriminatory cooperators benefit from the help of those around them. The result is that a region of discriminators tends to expand at the expense of an adjacent region of egoists (Axelrod, Hammond, and Grafen 2004:1834).

Although the theoretical focus of this particular model is specific to strategies of assortment among kin, the general principles can potentially be more broadly applied. In a similar simulation, Hammond and Axelrod (2006b) and Shultz, 
Hartshorn, and Hammond (2008) demonstrated that a strategy of in-group favoritism (or "ethnocentrism"), based on assortment of cooperators bearing like tags, outcompetes alternative strategies such as cooperating with neither in-group nor out-group ("selfish"), cooperating indiscriminately ("humanitarian"), and cooperating only with out-groups ("traitorous"). Mechanisms of limited dispersal (keeping offspring close) and viscosity of the environment ensured the eventual stabilization of in-group favoritism in about $75 \%$ of the population despite intense early competition from the humanitarian strategy. A further model demonstrated that tag-based strategies considerably enhance cooperation when dispersal is increased (compared to models based on dispersal alone) and when the benefit to cost ratio is reduced (Hammond and Axelrod 2006a).

Other recent assortment and tag-based and similarity-based approaches to the evolution of contingent altruism have considered the variable effects of different levels of genetic stability on the emergence and stabilization of cooperation (i.e., mutation rates: Hales 2005; Spector and Klein 2006; and tagstrategy coupling: Jansen and Van Baalen 2006), territorial and population structures (e.g., Antal et al. 2009; Colman, Browning, and Pulford 2012; Le Gac and Doebeli 2010; Lehmann and Perrin 2002; Spector and Klein 2006; Traulsen and Claussen 2004; Traulsen and Nowak 2007), environmental feedback (e.g., Pepper and Smuts 2002), tag (or "signal") complexity and diversity (Santos et al. 2011), socially acquired tags and gene-culture coevolution (Ihara 2011), n-person games and sociability of agents (Howley and Duggan 2010), sexual reproduction or "mixed marriages" (Lima et al. 2009), and availability of social information (Masuda and Ohtsuki 2007). Notably, at least three of these models have demonstrated the evolution of tag-contingent or similarity-based cooperation in a well-mixed population (i.e., no spatial structure is assumed and everyone is equally likely to interact with everyone else; Antal et al 2009; Colman, Browning, and Pulford 2012; Ihara 2011).

Some of these models have relaxed the assumption that contingent cooperators share and recognize identical and shared tags, exploring instead the viability of mechanisms that are sensitive to degrees of phenotypic similarity. Riolo, Cohen, and Axelrod (2001) demonstrated that tag-based cooperation arises between nonkin, and in the absence of memory of past encounters, when cooperative agents limit their cooperation to others who are sufficiently similar to themselves (determined by an individual "tolerance" quota). Although tags in their model are discrete, the approach can also be adapted to continuous "phenotypic spaces." In such scenarios, no two individuals would have exactly the same tag, but the conditional behavioral strategy would be triggered by sufficient phenotypic similarity (Antal et al. 2009:8599; Colman, Browning, and Pulford 2012). Costs of cooperation could also be calibrated by this measure, yielding a contextually (e.g., taskspecific) sensitive discrimination mechanism that titrates degrees of cooperativeness according to measures of self-sim- ilarity. Such a mechanism would preclude any prior necessity or assumption of bounded and stable groups within which identical tags are inherited and maintained. Rather, a mosaic of partially overlapping personal networks would emerge across neighboring individuals within a population, each determined by the self-proximity measure and behavioral strategies of the focal individual.

The possibility that similarity can breed cooperation potentially helps to explain the origins of pervasive biases guiding human sociality (e.g., homophilous friendship preferences) and cultural transmission (e.g., within-group imitative learning biases). An alternative and potentially competing account postulates that the psychological propensity to imitate and selectively interact with individuals who share one's symbolic markers has been favored in selection because it allows individuals to learn and adopt the right norms of the cultural group and thereby reap the fitness rewards of social life (Richerson and Boyd 2005:212-213). According to this account, the capacity for large-scale cooperation among strangers is the result of a selection history of within- and betweengroup competition (e.g., Boyd and Richerson 2009; Boyd et al. 2003). Within the group, an individual must acquire and follow group norms in order to reap the benefits of group living. Between groups, conquest is the reward of those whose norms bestow a group-level advantage in competition (Richerson and Boyd 2005). A coevolutionary helix of genetic and cultural selection for both norms and the psychological propensity to acquire the norms of one's group accounts for the proximate and ultimate causation underlying group-based trust, in-group favoritism, out-group dispreference and hostility, group marking, "tribal instincts," and inclusion and exclusion mechanisms (e.g., Boyd and Richerson 2009; Chudek and Henrich 2011; McElreath, Boyd, and Richerson 2003; Richerson and Boyd 2005).

Proponents of cultural group selection models have criticized tag-based approaches for failing to account for the emergence of discrimination mechanisms, modeling the effects of discrimination rather than accounting for its causes. Henrich (2004) directly challenged the assumption commonly built into these models of an a priori bias in favor of tag-marked cooperators and against defectors. The work of Riolo, Cohen, and Axelrod (2001), for example, demonstrated that cooperation could evolve if individuals with matching tags always help each other. But, as Henrich questioned, why should this bias be assumed (2004:138)? Where are the inevitable pretenders and defectors?

Subsequent tag-based models (introduced above) explored the conditions that support cooperation in the absence of such assumptions and the conditions under which discriminatory biases can evolve and be sustained. For example, Hammond and Axelrod (2006a) compared the individual and joint effects of a range of factors and parameters on the evolution of cooperation, including the presence and absence of tags and tag-based strategies (including defection), the costs of cooperation, and the population structure within which in- 
teractions occurred. Although no bias toward similar others was assumed, contingent cooperation emerged as the most common strategy in a model combining population viscosity (i.e., local, rather than random, interactions between individuals) and tags. The combined conditions of viscosity and tagmediated interaction allowed contingent cooperation to invade, spread, and resist invasion by egoists even when cooperation was expensive and population viscosity low (thus precluding reciprocity).

Theoretical debates and demonstrations notwithstanding, tag-based theoretical accounts of cooperation have largely failed to address the question of whether there are any human traits that could reliably serve to support the emergence of tag-based cooperation. Cultural group selection models lump all symbolic group markers together as part of a broader coordination mechanism: they "allow individuals to interact with others who share their social norms" (McElreath, Boyd, and Richerson 2003:123). According to these models, culturally transmitted tags help solve coordination problems among individuals, but they do not provide a reliable mechanism for the assortment of cooperators. The problem, according to Richerson and Boyd (2005:213), is that culturally transmitted symbols are easy to fake: "talk is cheap and so is hair dye" (although see Henrich and Henrich [2007:176] on the special properties of linguistic markers as reliable coordination guides).

It is not difficult to identify candidates for easy-to-fake tags within the symbolic cultural record. Cheap talk, hair dye, body paint, personal ornaments, dress styles, cuisines, badges, tattoos, and many other ostensive cues potentially communicate a lot about an individual, but, insofar as they are vulnerable to fraud, they are unlikely foundations upon which tag-based contingent cooperation could emerge. Discriminatory strategies that are based on easy-to-fake tags invite exploitation. Individuals who fall within the right tag-tolerance sphere of a discriminating cooperator, but who free-ride on the benefits of cooperation, destabilize cooperator-cooperation assortment, and this ultimately causes a sharp decline in cooperation in the population. Simulations have demonstrated that cooperation can recover as a new dominant cluster establishes, generating a long-term "tidal" pattern (referred to as "beard chromodynamics"; Riolo, Cohen, and Axelrod 2001; Sigmund 2009; Sigmund and Nowak 2001; Traulsen and Nowak 2007). Nevertheless, it is difficult to see how any fitnessbeneficial tag-based strategy could spread and dominate at all if tags could be readily donned and removed at will. Exploitation by defectors would be rife and cooperation highly risky. So, are there any human traits that could potentially form a robust foundation for the assortment of cooperators, allowing tag-based cooperation to emerge?

A reliable and evolutionarily viable tag makes a number of demands. It must be a property of the individual yet comparable across individuals, readily observable (by whichever sense), and hard to fake. Tag inheritance should be low-cost; a cheap-to-acquire and hard-to-fake tag will sustain a more favorable cost-benefit balance sheet than an expensive-toacquire and hard-to-fake tag. Further, the models described above have shown that tag-based strategies can rapidly generate dominant clusters of tag-sharing cooperators, but that these are highly susceptible to invasion by defectors who bear the same tag. Mechanisms that prevent tags from coalescing into an indiscriminable type and/or fine-grained signal decoding mechanisms that permit individuals to perceive subtle variation among individual tags could potentially mitigate against the frequency and extent of such invasions and maintain signal variation required to usefully guide cooperative strategies (Hales 2005; Ihara 2011). Plausible mechanisms include imperfect inheritance across generations, ontogenetic change, and cultural isolation and drift.

Finally, if tags played a part in the emergence and maintenance of cooperation in large, regional networks, the candidate tag should be evolutionarily ancient and cross-culturally universal. Insofar as the cooperative strategies of the young have important consequences for their fitness, we might also hypothesize the early emergence of tag production and discrimination in development, including the attentional and evaluative biases that a tag-based strategy entails.

Tag requisites and rationales are summarized in table 1 . Arguably, although no single requirement is absolutely necessary for the emergence of cooperation under every possible set of limiting conditions, and the satisfaction of all requirements is unlikely jointly sufficient to account for cooperation among strangers in large human societies, the existence of a human trait that meets all these demands would, at the very least, suggest that the possibility of tag-based cooperation has been too hastily dismissed in existing evolutionary accounts of human cooperation.

Recently, several researchers across a range of disciplines have claimed that linguistic cues inherent in speech accent, or patterns of intonation and phonology, harbor special potential as reliable tags for the orientation of social and cooperative preferences among strangers (e.g., Irwin 1987; Kinzler, Dupoux, and Spelke 2007; Kinzler et al. 2009; Nettle 1999a; Nettle and Dunbar 1997; Roberts 2008; Sigmund and Nowak 2001; Tooby and Cosmides 1989; Traulsen 2008). Despite the existence of a large body of relevant empirical literature, there has been no systematic evaluation of these claims against the evidence on language and on the known or hypothesized conditions of human evolution, or of whether linguistic cues meet the minimal criteria required for tagbased cooperation to emerge and be sustained. Thus, while biological theory continues to make progress of potentially considerable importance for our understanding of human social evolution, the relevance of these recent advances remains a matter of deeply uncertain conjecture. In light of the wealth of relevant sociolinguistic, historical, comparative, developmental, archaeological, anthropological, sociological, and neuroscientific research on language that now exists, a synthesis of existing theory and evidence is both possible and timely. The remainder of this paper aims to initiate this en- 


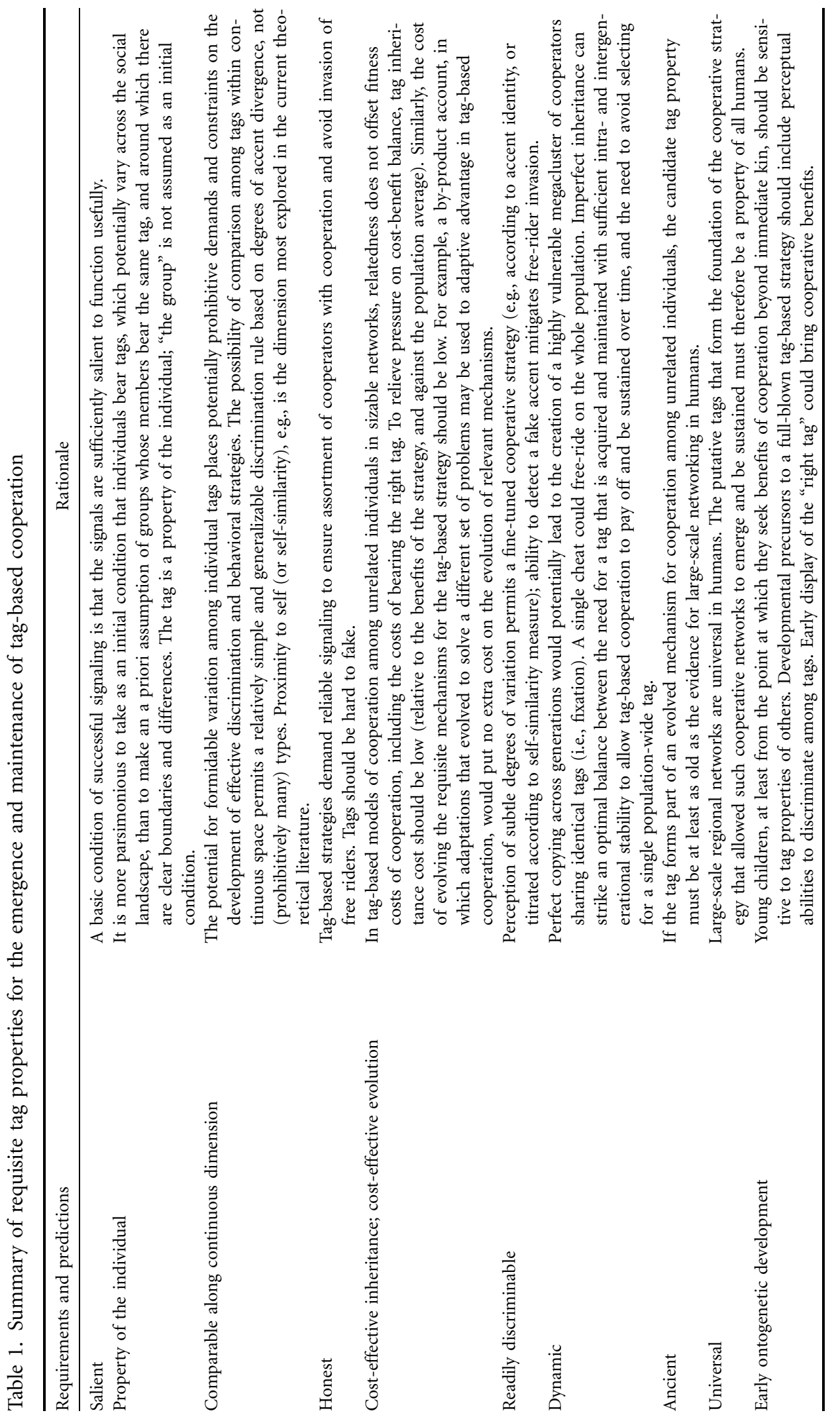


deavor and to appraise the possibilities for, and potential value of, a concerted program of research for the future.

The following section reviews literature relevant to each of the minimal demands summarized in table 1. In light of the growing number of claims about the suitability of language, the review will focus on the possibility that linguistic cuesparticularly accent-meet the demands of a viable tagging strategy.

Nota bene: although the specific focus here is on language and accent, this does not preclude the possibility that other forms of cultural marker fulfill some or all of the criteria below equally well, or that an aggregate tag or measure of self-similarity could underpin such a strategy. Heyes (forthcoming), for example, has recently drawn attention to the role of "dumb imitation"-imitation that does not depend on, nor is successfully achieved through, deliberate and rational calculation-in the faithful transmission and maintenance of group-specific cultural variations in gait and facial expression. Vocal imitation may be a specific, albeit highly sophisticated, type of "dumb imitation."

Further, the emphasis in this section will be largely on the properties of linguistic tags and the question of whether tags are fit for purpose, rather than on the form and prevalence of any tag-contingent behavioral strategies, and their possible selection history. We will return to these issues at the end of the section, and particularly in the section "The Evolution of Tag-Based Cooperation in Humans."

\section{Accent as Tag}

\section{Salient}

The human linguistic signaling system, defined by the uniquely flexible communication of propositional content via a shared system of symbolic representation, is a cornerstone of human cultural transmission and evolution. The evolutionary consequences of language for uniquely human sociality-including cooperation-are the focus of a large and growing literature (e.g., Brinck and Gardenfors 2003; Dunbar 1996; Tomasello 1999, 2008). The relevance of language for reputational mechanisms, for example, has been widely noted; language permits the transmission of forms of knowledge that would otherwise remain mute, including socially strategic information about the behavior of others (Smith 2003). Indeed, it has been argued that the evolutionary raison d'être of language is to facilitate social cohesion via the transfer of such information (Dunbar 1996).

However language emerged, it harbors valuable socially strategic signaling potential that can be realized irrespective of the semantic content of the message conveyed. Decades of sociolinguistic research have provided considerable evidence that the language a person speaks, and the particular variant that is spoken, is a salient guide to social identity (e.g., Ed- wards 2009). Accent is one of the most fundamental and salient social identity cues that language affords (Fuertes, Potere, and Ramirez 2002; Gluszek and Dovidio 2010). Two people may say exactly the same thing, but, depending on their accent, they may be perceived, trusted, evaluated, and heeded differently (e.g., Giles 1973). Although accent may not always be the most salient signal of social identity, the rapidity with which people can extract this information from accent cues, and the readiness with which it is used in guiding behavior, testifies to the salience of accent in the human linguistic communication.

The importance of accent as a social marker is powerfully illustrated in the use of modern-day Shibboleth Tests, named for the famous biblical pronunciation test in which the Gileadites detected and killed 42,000 fugitive Ephraimites by their inaccurate pronunciation of the word shibboleth. "Linguistic passwords" continue to be employed in a wide range of forensic contexts today, from identifying illegal immigrants to rooting out infiltrators in armed conflicts (Blommaert 2009; McNamara 2005). Although such tests are ethically and methodologically problematic, lacking some of the reliability of modern bureaucratic safeguards such as identity cards, finger prints, face recognition technology, birth certificates, passports, and so on, they nevertheless serve to highlight the salience and importance of accent in the absence of such accoutrements, and its affordances as a hard-to-hide trait.

\section{Property of the Individual}

Although linguistic cues such as accent and dialect can be used to socially "place" speakers, they are properties of individuals. Accent, dialect, and language are often considered to be "whole group traits" insofar as they are comprehensively and exclusively shared by members of a particular group (Foley 2004). But, even in those cases for which linguistic boundaries may be relatively well defined (e.g., between mutually incomprehensible languages), comprehensive and perfect parity rarely, if ever, characterize the form that language takes within those boundaries. Indeed, the variability within every sphere around which a boundary might be drawn persists down to the singular individual; linguists use labels such as "dialect," "sociolect," and "idiolect" to capture this reality at the levels of culture, subculture, and individual, respectively.

\section{Continuous (Comparable) Trait}

It is important to note, however, that linguistic variability is not randomly distributed. Idiolects are roughly nested within sociolects, dialects within languages, languages within language families, and so on. Two sociolects within a single dialect are likely to resemble one another more closely than two sociolects from two different dialects. Yet, the boundaries between these nested domains are notoriously difficult to draw. This is because, for the most part, language boundaries do not really exist. Trudgill (2000) explains as follows: "If you 
travel from Norfolk into Suffolk, the county immediately to the south ... you will find ... that the linguistic characteristics of these dialects change gradually from place to place. There is no clear linguistic break between Norfolk and Suffolk dialects. It is not possible to state where people stop speaking Norfolk dialect and start speaking Suffolk dialect. There is, that is, a geographical dialect continuum" (3).

This linguistic continuity allows for measurement of "linguistic distance" and the exploration of questions about the relationship between linguistic and geographic distances. Dialectometrists use a range of methods to calculate linguistic distance, including single-item analyses (e.g., the pronunciation of a particular word across a language area) and, more recently, aggregate-item analyses (e.g., the comparison of dozens of word-pairs across hundreds of sites; see Heeringa and Nerbonne 2001; Nerbonne 2010). Mean varietal linguistic distances between sites can be visualized on a regional map, represented, for example, by the thickness of the lines connecting each site to every other site. Questions can then be explored concerning the sufficiency of geographic variation to account for linguistic variation. A perfect continuum model, for example, would assume a perfect mapping between geographic distance and linguistic distance (allowing, perhaps, for the mediating influence of particular topographical constraints, such as mountain ranges and rivers, on population mobility).

Recently, the development of multidimensional scaling mapping techniques has provided the most sophisticated portrayals of linguistic variation to date for a range of languages. The map in Nerbonne (2010) indicates pronunciation distances for 186 word pairs across 20,100 pairs of German sites using intensity variations across a three-element color scheme. The result is a patchwork of color gradients gradually turning from gold in the southeast, through greens and reds in the west and east, to blues and ultimately lilacs in the far north. Nerbonne concludes that a dialect continuum that is compatible with the existence of areas but that denies the existence of sharp boundaries emerges saliently.

Linguistic variation thus affords a readily measurable tag of self-similarity comparison. The continuum of variation potentially permits gradual titration of the costs or kinds of cooperative interactions across linguistic distance (and ultimately across the boundary of mutual intelligibility). Crucially, its utility in this regard depends on how "mappable" this variation is for ordinary human perception and cognition, a point to which we return below (see the "Discriminable" section).

\section{Honest}

The value of linguistic varieties as tags guiding cooperation depends on whether these varieties can be readily faked. Much speculation about the reliability of linguistic cues as honest signals is based on personal experience, anecdote, and assumption. New words and dialectal expressions can be readily grasped and learned, but correct (i.e., local-like) pronunciation poses much greater challenges. Passing for a local beyond one's immediate accent area is notoriously difficult-whether attempting to speak a foreign language or native-language dialect. Certain features of one's habitual speech patterns can also be hard to hide, especially if distinctions (such as between $l$ and $r$ ) are no longer consciously accessible (see below). Actors receive intensive and immersive foreign and regional accent training to boost the credibility of their characters. Speech manuals, voice guides, and an increasingly wide array of YouTube videos promise to impart the secrets of speaking convincingly in the accent of one's choice. These point to the real challenges that faking an accent presents.

A large but divided literature on language acquisition has examined the causal basis of this phenomenon. Extensive debate has focused on the "Critical Period Hypothesis" (CPH) for second-language acquisition (Nikolov and Djigunovic 2006). The central claim of the $\mathrm{CPH}$ is that natural language acquisition (which, according to certain accounts, relies on plasticity of procedural memory and implicit competence) is available to young children, but not older children and adults. The plasticity of the mechanisms underpinning natural language acquisition is said to decrease with age. Older learners must therefore compensate for the resulting decline of implicit competence by recruiting explicit mechanisms, such as declarative memory and metalinguistic knowledge (see Nikolov and Djigunovic 2006). The critical period thus accounts for the difficulty that adult learners have in acquiring language with native-like proficiency. Several questions and controversies have dominated the research following from these claims: If there is a critical period, when does it end? Can adults ever attain native proficiency? To what domains of language does the critical period apply (e.g., pronunciation, grammar, vocabulary)? Is early learning subserved by different mechanisms to those that subserve late learning?

A huge $\mathrm{CPH}$ literature exists, and frequent reviews update the ever-shifting state of the art (e.g., Nikolov and Djigunovic 2006; Scovel 2000; Singleton 2002). Yet, because the hard-tofake-ness of linguistic cues does not rely on a strict reading of the $\mathrm{CPH}$, much of this research addresses concerns that are tangential to the question of tag reliability. For example, even if it is demonstrated that adult learners of a foreign language can achieve native-like proficiency in grammar and pronunciation, what matters for the success of the tag is the cost of obtaining such proficiency. Prolonged and intensive immersion is known to have positive effects on language learning, including the elimination of the beginner's telltale foreign accent (as judged by native speakers). Yet, this merely supports the claim that linguistic cues are hard to fake, even if they are not impossible to fake under the right set of conditions. It further suggests that without sustained exposure to the cue via direct social contact and integration with the local speech community, and opportunity for rehearsal, an individual would be unable to pass as a local.

In a population of tag-based cooperators, such a cost is 
simply prohibitive for the stranger seeking to operate a swift hit-and-run strategy. If bearing the right tag is the condition for access to the benefits of cooperation, and the only way in which the tag can be acquired is by being born into the community or through later long-term integration, the nonnative interloper faces an impossible task. What matters, then, for the honesty criterion is that achieving native-like proficiency in nonnative language is extremely difficult. Considerable research in linguistics now focuses not on whether this is so, but why (see Meltzoff et al. 2009).

\section{Cost Effective}

The vast literature on the purported critical (or "sensitive") period largely results from the perceived discrepancy in the mechanisms and costs of early first-language (L1) and late second-language (L2) acquisition. The challenges the adult faces in becoming proficient in a second language, dialect, or accent are thrown into sharp relief against the ease with which the child acquires her or his "mother tongue." One of the most fundamental challenges guiding language acquisition research generally has been to explain the remarkable facility with which young children acquire language (whether one language or more) or shift from one language or dialect to another (Tagliamonte and Molfenter 2007). The development of language in children is tellingly described as "natural." Whatever the proximate and ultimate causes and costs of this natural facility, once they are in place, the tag comes for free. After all, no one speaks without an accent.

Concerning evolutionary start-up costs, the burden of the emergence of accent tagging may have been entirely borne by an evolutionary process that dealt with a very different set of costs and benefits. This process ultimately led to the emergence of language and all the cognitive, motivational, and anatomical adaptations that this entailed. Again, accent tags may have hitched a ride for free on the back of variation ensuing from population dispersal and drift.

Most important for the tagging properties of accent and dialect is the capacity for vocal imitation (Fitch 2000). The accent that is inherent in the speech of every modern human is likely a by-product of the capacity to vocally imitate novel sounds. The specific evolutionary pressures that produced this capacity are a matter of debate, however, and this has prompted some interesting evolutionary hypotheses.

Clearly, humans' extremely fine-tuned vocal learning capacities allow for the transmission of extensive vocabularies. But was this the ultimate, evolutionary function of sophisticated capacities for vocal imitation? Some have suggested that extensive vocabularies capitalized on vocal learning capacities that had already emerged in a different context to serve a different function. Fitch (2000), for example, suggests that vocal learning may have originated as a device for generating complexity per se, a property that has obvious fitness consequences in birdsong and other animal vocalizations (e.g., whales and seals). Alternatively, where the value of group membership was high, fine-tuned vocal imitation may have been the mechanism that served to maintain reliability of vocal indicators as badges of group membership. An evolutionary arms race between group members and interlopers may have selected for sharper discrimination and increasingly complex "passwords," leading to runaway selection for increased vocal learning and finer perceptual tuning (Fitch 2000: 264).

This intriguing proposal effectively inverts the by-product account-rather than accent hitching a ride with language, accent potentially emerges prior to language. The independent pressures that selected for increasingly sophisticated and subtle tag signaling would ultimately have permitted the vocal communication of complex semantic structures, not the other way around. In this account, then, the sophisticated tag properties that we can observe in language today did not all come at once, nor did they come for free. Rather, they emerged out of an evolutionary arms race among competing groups. In contrast to the by-product account, tag selection in this armsrace account comes at a cost. The viability of this cost must ultimately be weighed in terms of the benefits of having a reliable group boundary patrol.

\section{Discriminable}

Whatever the evolutionary history of the vocal technology underpinning the controlled production of fine-grained speech sounds, tag-contingent cooperation demands discrimination abilities that at least match the production capacities that permit subtle variation. This serves not only as protection against the defection of others, but also, and perhaps more relevantly, it potentially allows a finely graded cooperative strategy. Decisions about degree of cooperation could thus be finely calibrated against incremental linguistic distance. But how well do we detect fake accents? And how proficiently do we discriminate the "true" accents of those around us?

A growing literature in forensic linguistics on accent and voice recognition accuracy is particularly relevant. Because accent is one of the first things forensic practitioners consider when establishing the identity of a speaker (Hollien 2002; Jessen 2007), there is a high premium for the criminal on successful imitation. Numerous experimental studies have shown that accent switches and disguises can fool listeners (e.g., Markham 1999; Neuhauser and Simpson 2007; Sjöström et al. 2006). It is important to note, however, that while listeners/judges are not always linguistically or professionally trained, speakers in these experimenters are often chosen because of their "conspicuously good pronunciation performance" (Markham 1999:292). Again, the relevant question for our purposes is not whether speakers, under the best possible conditions, can fool listeners, but whether the conditions that permit deception are reasonable under relevant evolutionary conditions. Nevertheless, even under biased laboratory conditions, well-developed accent awareness has a strong positive influence on detection. When their own local 
accent is disguised, native listeners readily identify nonnative characteristics, while listeners who are merely acquainted with the accent do not. These studies suggest that successful imitation demands considerable exposure to the target accent and that, even under these conditions, native listeners are readily able to detect telltale nonnative characteristics.

The second question regarding the discrimination of finegrained regional differences among native speakers may be further subdivided into two parts: How well do listeners perceive differences? How successfully do they extract information about social identity from these differences? The most relevant research on the perception of dialect variation tends to conflate these two questions within a discrete-trait classification paradigm. Typically, native speakers are asked to classify speech samples by region in a forced-choice design. Overall, studies have shown a strong effect of accent awareness (Clopper and Pisoni 2006). Geographically mobile individuals, for example, more accurately place speakers of different dialects than individuals who have exclusively lived in their native region. All individuals, it seems, know their own accent: local varieties consistently elicit higher levels of categorization accuracy. The greater the distance from their own accent, then, the more likely participants are to miscategorize accents (see Clopper and Pisoni 2007).

Finer-grained discrimination closer to home is exactly what we might expect from a nondiscrete tagging strategy that is premised upon self-similarity judgments and that is capable of being finely calibrated to relevant tag variation (e.g., among neighbors). There has been surprisingly little research on this subject, but one recent study provides clear results. Ikeno and Hansen (2007) compared the abilities of British English speakers, US English speakers, and nonnative English speakers to discriminate three regional British accents (Belfast, Cambridge, and Cardiff). British listeners performed with $83 \%$ accuracy, while US and nonnative listeners performed at around chance (56\% and $45 \%$, respectively). These results suggest, quite remarkably, that language parity is of little benefit to accent discrimination. Sensitivities appear to be tuned by experience according to (potentially fitness-related) local relevance.

More recent research using free classification methods with US English speakers and listeners suggests that when participants are given the option to freely classify talkers, they "make fine-grained distinctions between regional dialects" that forced-choice tasks failed to identify (Clopper and Pisoni 2007:435). Further, whereas in forced-choice studies listeners categorized geographically contiguous dialects together, free classification seems to be more firmly, and accurately, based on phonological contiguity. This provides a suggestive hint that listeners can accurately perceive and, under the right conditions, prioritize linguistic distance in accent classification tasks. New methods of elicitation and statistical analysis (e.g., clustering and multidimensional scaling) potentially permit a range of relevant experimental designs that could shed more light on discrimination abilities, particularly the ques- tion of whether (and at what scale) listeners spontaneously make finer discriminations between local varieties than between nonlocal varieties (see Clopper 2008).

\section{Dynamic}

The variation within and across languages that permits tagbased discrimination owes, ultimately, to the genetic underspecification of language. Language is supported, acquired, and maintained by a combination of biological and cultural mechanisms, and variation is primarily, if not wholly, a product of complex processes of cultural evolution (but see Dediu 2011; Ladd, Dediu, and Kinsella 2008). How much variation can be explained by cultural isolation and drift, population and demographic factors (e.g., language contact, community size), active selection on the part of individuals (i.e., "social selection"; Nettle 1999a, 1999b), and imperfect learning has been the subject of much research in historical, sociological, and evolutionary linguistics (see Chambers, Trudgill, and Schilling-Estes 2002; Trudgill 2011). This is a vast literature, and any review of the wide range of findings is well beyond the scope of this paper. It is widely agreed that linguistic variation and change are associated with a range of factors influencing acquisition across generations and maintenance within them.

Although the existence of variation is not in doubt, it is less clear that the amount of variation and the rate of change are viable for the emergence and maintenance of an accentmediated cooperation strategy. The value of linguistic signals as reliable indices of cooperative potential relies on striking a viable balance between tag stability and change. If successful tag-based cooperation evolves and spreads throughout the whole population, the system becomes vulnerable to wholesale invasion by free riders. Further, because zero signal variation means zero discrimination, tagging no longer affords a mechanism for the fine-tuned calibration of cooperative behavior-an argument that has been used to support the claim that, historically, linguistic cues may have more usefully guided cooperative decision making than the comparatively invariant markers of race (Kinzler, Corriveau, and Harris 2011; Kurzban, Tooby, and Cosmides 2001). Too much random modification, however, jeopardizes assortment, bringing the same consequences as too much tag faking. Mutation in "the wrong direction" (i.e., away from any potential source of cooperation) can threaten the stability required for cooperators to assort with cooperation. In a large group of mobile individuals operating a tag-based contingent cooperation strategy, some degree of tag stability is necessary to ensure that cooperators assort with cooperation.

Incremental change, rather than "punctuational bursts," could help to sustain an optimal mutation rate without jeopardizing assortment. Alternatively, or in addition, active selection among available or new variants by individuals and groups ("social selection mechanisms") could channel incremental mutation along individually advantageous routes (see 
Atkinson et al. 2008; Nettle 1999a). In such scenarios, individuals actively engineer change to align more closely with prospective cooperators. Simulations inspired by recent models of language change could potentially identify rates of stability and mutation for the evolution and maintenance of tagbased assortment strategies (e.g., Baxter et al. 2009; Nettle $1999 b$ ). These could then be scrutinized against real-world patterns documented in historical and evolutionary linguistics on language variation and change as well as insights from studies on "dialect leveling" (or convergence) toward regional and national standards. There is considerable scope for empirically informed simulation work on these issues.

\section{Ancient}

The ancient emergence of accent is not a design characteristic but rather a precondition of accent tagging. If accents formed part of an evolved mechanism for cooperation among unrelated individuals, then they must be at least as old as the evidence for large-scale networking in humans.

The question of how old accent tags are depends on whether standard linguistic variation (in the sense used in modern linguistics) or variation in complex prelinguistic vocalizations is taken to be the relevant property on which tagbased cooperative strategies emerged. In principle, full-blown language is not a necessary precondition of accent tagging. Indeed, the social identity/identifying functions of withinpopulation variability in animal vocalizations (broadly construed) is the subject of much current research across a range of species, including killer whales (Yurk et al. 2002), bottlenose dolphins (Janik, Sayigh, and Wells 2006), wild chimpanzees (e.g., Herbinger et al. 2009), greater spear-nosed bats (Boughman and Wilkinson 1998), Mexican jays (Hopp, Jablonski, and Brown 2001), yellow-naped Amazon parrots (Wright and Wilkinson 2001), and European starlings (Hausberger, Bigot, and Clergeau 2008).

Linguistic tags have probably therefore been around for as long as, if not longer than, humans have required them in tag-based discrimination. Population dispersal, isolation, and drift would have generated linguistic variation through incremental change. With the increases in population dispersal from the African Middle Stone Age (MSA) onward, which opened up possibilities for fitness benefits and costs on a larger geographic and social scale, this variation could be exploited to serve a tagging function and guide cooperative decision making (McBrearty and Brooks 2000; see following section). Insofar as social selection processes channeled variation and intensified the pace of change, we might expect some increase in the rates of local cultural evolution in nonlinguistic domains also (Powell, Shennan, and Thomas 2009; Shennan 2001). The material record studied by archaeologists provides potentially informative evidence, or a proxy indicator, of the importance of linguistic varieties as social tags in MSA and Middle-to-Upper European Paleolithic society.

The most persuasive evidence of materials that were crafted and used as markers of social identity is standardized beads and body ornaments. Kuhn and Stiner $(2007 a$, 2007b) have argued that the emergence of simple, standardized, durable forms of body ornamentation in the form of beads, shells, and teeth testifies to a new medium for signaling social information. In contrast to body decoration, which might have been employed, for example, in the service of attracting mates or intimidating adversaries, the appearance of ornamentation in the material culture of the earliest anatomically modern Homo sapiens marks a development in social communication technologies that reflects "a burgeoning need to communicate with stranger and friend alike" (Kuhn and Stiner 2007b:47). The material record dates the emergence of ornamental beads to at least 65,000-75,000 years ago (Blombos Cave, South Africa). McBrearty and Brooks (2000) further review evidence for comparable symbolic artefact forms from the African MSA that predate those of Europe by tens of thousands of years. Signs of long-distance exchange networks and the presence of stylistic provinces also appear earlier in the MSA, leading to the speculation that MSA groups signaled their identities as intensively as later, more crowded populations (McBrearty and Brooks 2000:531).

A survey of the geographical distribution and association of European Upper Paleolithic personal ornaments supports this interpretation of ornament use on a broad scale. Seriation, between-site clustering, and contour mapping of 157 ornament types sourced across 98 western European sites suggest long-established cultural differences among adjacent groups inhabiting the region by between 37,000 and 28,000 years ago (Vanhaeren and d'Errico 2006). In line with linguists' conceptualization of neighboring dialects as occupying positions on a continuum, along which adjacent but not distant dialects are mutually intelligible, the two most regionally distant beadtype "macrosets" do not share any bead types, but both share types with the intermediate macroset. Vanhaeren and d'Errico (2006) espouse more than an analogical link, however, suggesting that the identified sets and macrosets reflect ethnolinguistic diversity at the levels of language and language family, respectively (1119).

Kuhn and Stiner (2007b) argue that changing demographic conditions likely increased the frequency of interactions among strangers and that this, in turn, may have "fostered heightened sensitivity to group boundaries as a means of delimiting and defending territories" (48). That social boundary demarcation and territorial defense followed swiftly on the heels of social identity communication is debatable in light of characterizations of early human social organization as fluid, and "group structure" as "a convenient shorthand for the summation of intersecting networks, based on the individual and represented by a variety of geographical scales" (Gamble 1998:441). It is broadly agreed, however, that these objects were employed to communicate messages to individuals in "the middle distance" (Kuhn and Stiner 2007b:47) far enough that the message actually communicated new information but not so far that the code by which it was com- 
municated was completely unintelligible to the receiver. Analysis of raw-material transport distances suggests that some of the earliest supralocal communication and exchange networks are responsible for shifting artifacts as far as $300 \mathrm{~km}$ from their source (Marwick 2003). The MSA social landscape, from the point of view of any individual, was therefore "extended beyond either a foraging or subsistence scale" (Gamble 1998: 443) and had the symbolic tools that permitted and regulated large-scale, complex sociality.

The archaeological evidence briefly summarized here points directly to the early emergence of cultural artifacts that may have served to signal information about individuals and their social identities and indirectly supports the hypothesis that linguistic tags were salient features of these identities (Marwick 2005). The extent of subtle variation in accents in ancestral social landscapes is obviously difficult to establish. Nevertheless, research in sociolinguistics, historical linguistics, and dialectometry can potentially help to identify the geographical and population factors that significantly influence accent diversity and change. These general principles could be applied to the archaeological record to better infer patterns of linguistic variation and change from the available material signs of emergent large-scale sociality.

\section{Universal}

Accent tags are as widespread as language and may therefore be construed as universal (barring certain speech and hearing pathologies). Although people commonly claim that they do not have an accent, nobody who produces spoken language speaks without an accent.

Note that this egocentrism possibly hints that linguistic tag discrimination commonly operates a self-similarity modelif tag variability is measured using the self as reference point, then only distinctive features measured in relation to that baseline would become salient in perception and relevant in assessment. Other baselines might be regularly used, including regional and national "standards" (that, in certain cultural contexts, may be overrepresented in modern communications media, such as television and radio). Yet, even when the national standards of modern societies are associated with higher social class, the capacity for detection and comparison of difference can potentially still have important fitness consequences. In this case, accent variation would map onto variable socioeconomic status and access to resources.

\section{Acquired Early}

As with ancient emergence (see the "Ancient" section), the acquisition of core elements of tag-based cooperation early in ontogeny is not a minimal criterion or condition of an evolved tagging strategy. Nevertheless, if cooperative strategies based on linguistic tags have played a critical role in the emergence and maintenance of large-scale cooperation in humans, including young humans, we might expect to find some com- ponents of a tag-based strategy appearing early in development. The cognitive processes underlying the acquisition of the tag, and signaling and discrimination abilities, unlikely emerge all at once in the mature adult.

Because accent acquisition piggybacks on natural language acquisition early in ontogenetic development, it is unsurprising-perhaps inevitable-that accent tags appear early. A recent study investigating the acoustic structure of newborns' cry melodies is one of the first to provide evidence suggesting that neonates' vocal imitation skills are sufficiently developed to allow them to produce melody contours similar to those perceived in language prenatally. Reflecting the intonation patterns observed in their respective languages, French newborns tended to produce rising melody contours while German newborns more often produced falling contours (Mampe et al. 2009). By 10 months, there are patterned differences in the babbling of infants raised in different countries (de Boysson-Bardies 1993), and "mother-tongue" mastery is complete by 8 years of age (Ferguson, Menn, and Stoel-Gammon 1992). Whatever the functional or proximate explanations for precocious vocal learning capacities, the advantages for a tagging strategy based on the acquisition of local linguistic variants are obvious. It may be argued either that tags begin to emerge long before they are useful in cooperative interaction, or that "this behavior enhances the likelihood of mutual caregiver-infant affiliation" (Cross 2009:1079). Production is only one element of a tag-based strategy, however. Discrimination and appropriate tag-mediated behavior are also necessary.

Considerable attention has focused on infants' and young children's capacities to perceptually discriminate linguistic distinctions. Newborns and very young infants are capable of discriminating phonemes, language rhythm, and prosody (Eimas et al. 1971; Mehler, Jusczyk, and Lambertz 1988; Nazzi, Floccia, and Bertoncini 1998; Nazzi, Jusczyk, and Johnson 2000). Further, although infants begin life with the capacity to distinguish all speech sounds, these universal capacities are already language-specific by 12 months of age. Kuhl et al. (2006) compared American and Japanese infants' abilities to discriminate the American $l-r$ contrast. At 6-8 months, there was no difference between samples. By 10-12 months, American infants' performance had improved while Japanese infants' performance had declined.

The capacity to discriminate among sounds in natural speech likely provides some foundation for the ability to discriminate among speech cues that provide indexical social identity information, such as accent. Nazzi, Jusczyk, and Johnson (2000) present evidence that 5-month-old North American infants can discriminate not only between nonnative languages of different rhythmic classes (Italian vs. Japanese), and between US English and a nonnative language of the same rhythmic class (Dutch), but also between native and nonnative accented English (British, first language). Regional accent categorization tasks with older children have produced mixed results, however. French 5-6-year-old children were 
significantly better at classifying voices when distinguished by a foreign (second language) rather than a regional accent or by idiosyncratic voice differences (Girard, Floccia, and Goslin 2008). Another set of studies comparing British 5- and 7year-olds showed good foreign (but not regional) accent discrimination among the older children only (Floccia, Butler, and Goslin 2009). Discrimination capacities were also associated with exposure to greater dialectal variation in the family home.

A recent series of studies using a different methodology corroborates findings that foreign-accent discrimination emerges early and that it is a significant and salient influence on social behavior. This research is relevant not only to our immediate question of tag suitability and utility, and the ontogenetic development of relevant production and perception abilities, but also to broader empirical questions of whether and how accent-tag-mediated cooperative strategies actually work on the ground. Whereas decades of sociolinguistic research with adults has demonstrated the influence of accent on social evaluation in adults (e.g., Alford and Strother 1990; Bresnahan et al. 2002; Dailey, Giles, and Jansma 2005; Dixon, Mahoney, and Cocks 2002; Feldman 1968; Giles 1973; Milroy and McClenaghan 1977), only recently have developmental psychologists begun to conduct systematic research on how accent guides children's social preferences, evaluation, and cooperation (though see Day 1980 for a pioneering demonstration of Hawaiian children's accent-guided social preferences). Initial investigations have produced some striking results.

Research by Kinzler and colleagues (Kinzler, Dupoux, and Spelke 2007; Kinzler et al. 2009) found that infants and young children showed visual and social preferences for speakers of their native language. Five- to 6-month-olds showed a looking preference for native-language speakers over foreign-language speakers and native-language speakers who had a foreign accent, 10-month-olds preferred to accept a toy from a nativelanguage speaker, and 5-year-olds preferred to be friends with children who were native-language speakers and native-accent speakers (over foreign-language and foreign-accent speakers; Kinzler, Dupoux, and Spelke 2007). A series of follow-up comparisons and controls showed that accent is more important than race in determining children's friend choices and that this is not due to the relative familiarity of each dimension; children showed a preference for distorted faces paired with a native accent over typical faces paired with a foreign accent. Yet, in the absence of any linguistic signal, that is, when the target children were silent, participants selected typical faces over distorted faces (Kinzler et al. 2009). A separate study (Kinzler, Corriveau, and Harris 2011) demonstrated that 4-5-year-old children selectively learned from native-accented speakers over foreign-accented speakers, even when both speakers produced semantically uninformative and unintelligible speech (jabberwocky, or nonsense speech).

In summary, there is considerable evidence that accent meets the criteria of a reliable tag for the orientation of co- operation strategies in humans. Sociolinguistic research attests to the salience of accent in social interaction. Dialectological research and dialectometry bear out a continuity model of accent variation. Language acquisition research provides evidence that accent is cheap to acquire in childhood but hard to fake in adulthood. A by-product account puts no cost on the evolutionary emergence of accent-accent production, discrimination is the inevitable result of vocal imitation capacities, and variation is the inevitable result of cultural processes of isolation and drift (possibly helped by social selection processes). These processes potentially serve to sustain the variation required for accent tagging to remain a viable mechanism-guiding cooperation. Finally, tags are both ancient and universal, and the foundations of accent-based cooperative strategies are laid down from the first days of life.

The developmental literature reviewed above provides the first evidence that accent is a robust and salient guide to earlydeveloping social preferences. The apparent priority of accent over other salient markers of social identity so early in development has prompted the proposal that "accent is a privileged guide to cultural learning" and that "social preferences and reasoning based on accent may have origins in cognitive evolution"; specifically, researchers have speculated that "cognitive evolution may have favored attention to accent over other social variables (e.g., race) that would not likely have differed across neighboring groups in ancient societies" (Kinzler, Corriveau, and Harris 2011:110).

Systematic research across variable social-developmental and cultural contexts is currently being conducted to substantiate these claims (Kinzler, Shutts, and Spelke 2012). In light of the growing evidence that accent is a privileged guide to social evaluation among children (over and above other pillars of children's social worlds already well established in the literature) and that accent meets key criteria of a reliable and viable tag for the emergence and maintenance of cooperation, these evolutionary proposals merit some consideration. What evidence is there that conditions in human prehistory might have required and promoted the emergence of accent-tag-based cooperation, or cultural-tag-based cooperation generally? Below I sketch one possible but necessarily speculative account.

\section{The Evolution of Tag-Based Cooperation in Humans}

The preceding sections combine recent modeling work in theoretical biology and a range of empirical work relevant to assessing the viability of accent as a tag for the mediation of cooperation. This exercise is only interesting and useful to the degree that it produces a logically and empirically coherent account that can be situated within the history of human evolution. Earlier attempts (e.g., by Nettle and Dunbar 1997) to model the coevolution of linguistic social marking and cooperation were dismissed on account of the markers' unreliability. The relevant literature reviewed above considerably 
weakens this critique. A critical question remains, however: when in human evolution might tagging have become a relevant mechanism of assortment among cooperators?

It is generally agreed that tag-based mechanisms are useful when agents have no explicit knowledge of kinship and are unable to observe, gain information about, or remember others' actions (Riolo, Cohen, and Axelrod 2001; Spector and Klein 2006). Within and among small-scale residential groups of the earliest hominins (after our lineage diverged from the great ape lineage 5-7 million years ago), kin selection and reciprocal exchange (via capacities for memory and recognition) likely went a long way in regulating the assortment of cooperators (Silk and Boyd 2010). With increasing community size-from nonhuman primate-like bands to the large dispersed communities of Homo sapiens of the MSA (McBrearty and Brooks 2000)-keeping track of the behavioral records of all individuals (across all relevant interactions and partners) and any contextual information relevant to the evaluation of their behavior (e.g., kin relationships, interaction history) would have become increasingly difficult (Dunbar 2008, 2009; Layton and O’Hara 2010).

Large group size (Aiello and Dunbar 1993; Bocquet-Appel and Demars 2000; Bocquet-Appel et al. 2005; Dunbar 2008, 2009; Grove 2010a) intensified pressure on the sustainability of community cohesion (Grove 2010b; Milton 1999). An increasing reliance on meat as part of a high-quality diet that fueled hominin brain enlargement heightened the risks of resource scarcity - compared with gathering plant foods, success in hunting is considerably less predictable and more variable (Kaplan, Hill, and Hurtado 1990). These pressures increased further still with migration into poorer ecological zones at higher latitudes (Layton and O'Hara 2010). As a consequence, large communities would have been forced to disperse in order to meet daily consumption demands (Grove 2010b).

Although fissioning into smaller hunting and residential bands was necessary, there were still benefits of maintaining links at the level of the wider population and of forging links across vast areas and multiple habitat types (Cavalli-Sforza and Hewlett 1982; Hewlett, Vandekoppel, and Cavalli-Sforza 1982; McBrearty and Brooks 2000; Mellars 2005). Seasonal and other less predictable fluctuations in local resource availability, and disputes within bands, sustained the relevance of the regional community as a critical space within which cooperative exchange and migration could occur (Kaplan, Hill, and Hurtado 1990; Kaplan et al. 1985; Whallon 2006). Yet, such an insurance mechanism would have been wide open to exploitation. Population growth, dispersal, and migration all present challenges to assortment systems hitherto constrained by kin recognition or limited dispersal, and direct reciprocity, and greatly increase the chances that the resulting potential for anonymous interactions will be exploited by free riders.

Thus, even while large group sizes, enhanced hunting techniques, and opportunities for mobility and dispersal all brought opportunities for beneficial cooperative interaction and networking, the challenges of directing cooperation to the right individuals would have far surpassed the capacities of existing cognitive and social mechanisms for the effective tracking of individuals (Dunbar 2008). Secondary insurance mechanisms were required to sustain the assortment of cooperators with cooperation. Under these conditions, selection may have strongly favored the emergence of "discriminate sociality," or cooperative strategies based on a culturally acquired tag that provides reliable information about an individual's cooperative potential (e.g., Ihara 2011). In those situations in which cooperation with strangers could significantly affect fitness, individuals with the cognitive skills and motivations on which tag-contingent cooperation depends would potentially have had a fitness advantage relative to noncooperators and indiscriminate cooperators.

For the sake of further speculative argument, let us grant preliminarily (a) the problem of assorting cooperators with cooperation in large-scale, disperse societies; $(b)$ the logical plausibility of a solution based on cultural tags or self-similarity; and $(c)$ the existence of a reliable cultural trait, or set of traits, acquired via species-general learning mechanisms (such as [vocal] imitation mechanisms). How, then, did the evolved mechanisms emerge or come to be exploited within such conditions? Below, I sketch three general accounts, though this rather simplifies the full range of possibilities.

Account 1. The tag-based system is phylogenetically specialized. The tripart system-a specific tag (such as accent), tag recognition and discrimination, and tag-contingent cooperation-was biologically selected for due to the fitness advantages conferred on those bearing the strategy.

Account 2. The components of the specialized tag-based system are by-products of existing evolved mechanisms. The mechanisms underpinning these components were already in place but came to be conjointly exploited under changing demographic circumstances: for example, the individual acquires the tag through existing cultural transmission mechanisms (e.g., language-acquisition mechanisms), tag recognition and discrimination are sustained through existing general learning mechanisms (e.g., perception and attention attuned via early imprinting on caregiver's speech), and tagcontingent cooperation emerges as a by-product of existing mechanisms of discriminate cooperation (e.g., nepotism) and positive conditioning (e.g., on familiar speech sounds).

Account 3. The tag-based system is ontogenetically specialized. As in account 2, the components of the system were not biologically selected for their fitness benefits on the bearers of the tag-based strategy but are rather by-products of existing mechanisms now operating together within an enlarged scale of social interaction. Unlike accounts 1 and 2, however, the system is not necessarily biased toward a particular tag (such as accent). Rather, the tag exploited by the system is contingent on relevant environmental variation and is tuned during development. Relevant tag variation in modern societies may not match the relevant variation that guided tag-based strat- 
egies for most of our species' past. For example, under certain modern demographic circumstances, racial variation may serve as a more salient and useful guide to assortment than accent variation, even though exposure to race variation is a historical novelty (see, e.g., Bar-Haim et al. 2006).

Prior assertions about the tagging potential of accent have focused chiefly on the possibility of a phylogenetically specialized, biologically evolved system (in line with account 1 above). Alternative accounts 2 and 3 present a potentially more parsimonious argument. Mechanisms entailed by accent tagging may have readily co-opted and built upon existing mechanisms, such as the ability to detect self-similarity and to condition cooperative behavior according to the identity of the prospective social partner. It seems plausible that these component elements of a cultural-tag-based strategy have their roots in already established kin selection mechanisms. Further, as discussed above, the ability to produce and discriminate among accent tags may also have already been established by language (or proto-language) acquisition mechanisms. Indeed, the emergence of accent tagging is not necessarily contingent on the prior emergence of full-blown language but may be supported by largely extant capacities for vocal imitation and discrimination (which has apparent homologies in nonhuman primate species, e.g., Herbinger et al. 2009). By some accounts, then, the emergence of tag-based assortment demands few novel conditions or mechanisms, yet the effects are far reaching (Hammond and Axelrod 2006a, $2006 b$ ). Components of the relevant proximate mechanisms might have been largely in place, ready to exploit the accent variation already existing in the wider population of nonrelatives who were increasingly coming into contact with one another.

Language, of course, afforded unprecedented access to information about third parties and was therefore likely an important component of evolving contingent cooperation strategies. An account that situates the emergence of accenttagging strategies after the emergence of language would therefore have to account for the limitations of reputation in guiding assortment beyond the local group. As noted above, one obvious limitation is that reputational information is only as good as it is accurately obtained and stored in memory. Accent tags can potentially outcompete reputational information in terms of both reliability and cognitive economy. Or, as one reviewer of this paper also pointed out, both mechanisms could potentially work productively in tandem-individuals with relatively similar accents are more likely to have mutual acquaintances than people with relatively different accents, and this can potentially deter noncooperation.

But why should a cooperative strategy emerge on the basis of honest information about childhood origins in particular? One possibility is that origin tags provided a probabilistic signal of relatedness. As Dunbar (1999) states, "dialect will quickly become important as a badge of relatedness whenever dialect acquisition is based on learning from those individuals with whom you live at a critical early period of development (i.e., well prior to social independence)" (203).

Alternatively, the tag's capacity to reliably assort like with like regardless of relatedness or origin is potentially sufficient to sustain viable cooperation in large, dispersed groups: "the individual behavioral strategy which may be summed up as 'sound like those you wish to cooperate with, and cooperate with those that sound like you' is likely to be evolutionarily stable over a much wider range of circumstances than a 'cooperate with anyone' strategy" (Nettle 1999a:222), or a defect with anyone strategy (Axelrod, Hammond, and Grafen 2004). Recent modeling work has shown that culture-dependent discriminate sociality can be selectively favored even when tags constitute arbitrary cultural variations (Ihara 2011). Thus, there may be no logical necessity for the requisite tag to advertise properties of the bearer (other than that the bearer will cooperate with an individual with a similar tag). This does not preclude, however, that accent tags, which by their nature offer clues to early social origins, could be supplemented with additional expectations on the part of discriminators about behaviors and knowledge commonly associated with those origins (e.g., dialectal expressions, locally relevant information).

Language potentially feeds back into the evolutionary equation here through the labeling and reification of accent variations as linguistic groups and social categories. These reifications may be actively used to promote (illusions of) intragroup stability and intergroup difference. Nettle notes "far from using language simply to communicate information in an optimally efficient way, people use it to create and maintain social identity and social boundaries" (1999a:221). Considerable anthropological, sociological, and sociolinguistic research demonstrates the power of such discourse to mobilize group-level sentiment and intergroup bias (see Hadzibeganovic, Stauffer, and Schulze 2008 for a modeling approach to the coevolution of political and linguistic states). An interesting further consequence of categorization of accent variation into distinct groups is that stereotypical inferences and predictions about the members of those groups can emerge and be drawn upon to guide behavior. It is not hard to imagine that these stereotypes could bear upon perceived cooperativeness-for example, northern-accented people are friendlier than southern-accented people-and potentially come into conflict with the inferences delivered by tagging mechanisms based on self-similarity (e.g., if one's accent is closer to "southern" than "northern").

It is not necessary, then, to posit that "social speciation" emerges and stabilizes via psychological and social mechanisms that primarily serve, or explicitly function, to maintain group boundaries (cf. Richerson and Boyd 2005). Cooperation may be focused within a particular "group," but this pattern may emerge as the aggregate product of individual strategies for fitness maximization through reliable and successful assortment with the cooperation of others. Other individuals represent more or less promising possibilities for 
beneficial cooperation, and some cooperative partnerships and networks may be sustained over many iterations. Thus, rather than assume an initial social landscape characterized by stable and discrete in-groups and out-groups and "tribal instincts" (Richerson and Boyd 2005), tag-based approaches are grounded in the more realistic premise of tag-sensitive individuals discriminating among networks of scale (Gamble 1998; Palmer, Fredrickson, and Tilley 1997). If circumstance demanded it, such individuals could do well to extend their tolerance range to include the odd-sounding neighbors from the other side of the mountain range, or, when there is competition for scarce resources, to shrink their tolerance range to exclude their chief rivals.

This is not to argue that group-level selection processes are absent in human societies, historically or presently. Although mechanisms that serve to strongly maintain isolation between stable groups are not required for accent-mediated strategies to arise, it is not difficult to see how a tag-based strategy would rapidly lead to some degree of social speciation under even very loose migration constraints (e.g., due to physical or other ecological barriers). The resulting drift among tags would rapidly reduce the likelihood of cooperation across boundaries - the tolerance and exclusion rules of the tagbased strategy, added to the barriers to migration that generated the variation in the first place, would force populations apart (Riolo, Cohen, and Axelrod 2001). Deep divides and sharp lines would emerge, generating, in their turn, barriers to mutual intelligibility and communication.

In this way, tag traits can cluster in space and thereby come to be employed and perceived as markers of collective (group) identity. Such speciation turns tags into self-enforcing stereotypes "making it hard for tolerance to cross the divide" (Sigmund and Nowak 2001:405; see also Castro and Toro 2007). The ability to discriminate among tags beyond the divide declines as a function of reduced exposure, leading to othertag homogenization (e.g., Ikeno and Hansen 2007; Sangrigoli and de Schonen 2004). These conditions may have set in train a coevolutionary track that adapted and honed the intergroup biases and behaviors so readily triggered in laboratory conditions today. For example, although accent may be among the most salient tags guiding young children's social preferences (e.g., Kinzler et al. 2009), a wealth of social psychological research has shown that social behavior is guided by discrimination across a wide range of possible traits. Markers of difference, including easily acquired ones, are sufficient to activate in-group favoritism (Billig and Tajfel 1973; Tajfel 1982). Furthermore, many cultural artifacts can be perfectly cloned, providing superstimuli to systems that are sensitive to fine-grained degrees of difference and always on the lookout for resemblance (cf. Sperber and Hirschfeld's [2004] idea of proper and actual domains).

In summary, according to this tag-based account, and contrary to the prevailing assumption that all cultural markers (e.g., badges, flags, cuisines, dialects, accents) are equally unsuitable as foundations for the evolution of tag-based co- operation (McElreath, Boyd, and Richerson 2003; Richerson and Boyd 2005), accent may have delivered a viable, costeffective, and reliable by-product solution to the problem of assortment in large, mobile, dispersed groups. The reliability of accent tags may have mitigated the potentially harmful consequences of fallible human memory within the fissionfusion/aggregation and dispersal dynamics of early human groups. A combination of frequent reunion with opportunities for information exchange and group bonding (Gamble 1983; Whallon 2006) and accent marking may have met the challenges of maintaining viable cooperative relationships in large cooperative networks.

\section{Conclusion and Future Directions}

Do tag-based strategies-specifically, accent-tagging strategies-constitute an evolved mechanism guiding the assortment of cooperators with cooperation in human populations? Testing evolutionary hypotheses satisfactorily, particularly about the function and phylogeny or history of a proposed adaptation, is fraught with difficulty. The demonstrated logical possibility of tag-based cooperation as an evolved assortment mechanism, the plausibility of accent as a candidate tag, and the existence of cognitive mechanisms that permit and promote accent tagging in humans today are insufficient evidence by themselves of a phylogenetically specialized adaptation (as in account 1 above). A particular mechanism's current utility does not necessarily indicate ancestral function-the function of the mechanism may have changed over time (exaptation), or the mechanism may be better explained as a by-product of some other adaptation (as in account 2).

One of the most promising methods for generating increasingly precise predictions about how and why a particular human capacity evolved is to compare the human problem (the evolution of fitness-beneficial cooperation with strangers) and the conditions for evolution of the proposed solution (tag-based contingent cooperation) with comparable scenarios from other species. This comparative evolutionary method is established on the premise that relevant data from other species (that display similar problems and/or solutions) provide informative reference points for identifying the changes that needed to occur in human evolution for tag-based cooperation to emerge, and the selective advantages that this strategy afforded.

Currently, there are several promising candidates for comparison, particularly avian calls (e.g., Berg et al. 2012; Hopp, Jablonski, and Brown 2001), dolphin, whale, and bat vocalizations (e.g., Boughman and Wilkinson 1998; Ford 1991; Janik, Sayigh, and Wells 2006), and zebrafish pigments (Engeszer, Ryan, and Parichy 2004). To date, most of this exploratory and experimental research has largely focused on establishing how apparent tags are acquired and how they guide social behavior (e.g., aggregation and affiliation) — analogous to the vast body of research in sociolinguistics on the social utility of accents. For example, Engeszer, Ryan, and 
Prichy (2004) showed that adaptive shoaling behavior in zebrafish is guided by pigment preferences that are acquired through early social experience. The functions of such tag variants and tag-contingent behavior, if any, remain the focus of considerable debate (on vocal dialects in birds, e.g., see Feekes 1977; Goodfellow and Slater 1986), though it is widely suggested that socially acquired tags and preferences are particularly important in highly mobile social species characterized by fission-fusion dynamics. Referring specifically to avian linguistic diversity, for example, Hausberger, Bigot, and Clergeau (2008) suggest that multiple dialects may be useful in complex social situations, with scales of variation helping to form and maintain nested groupings that in turn reflect different geographical scales (680). Given the similarities between-and infancy of - the human and nonhuman theoretical projects on the role of tags in the evolution of cooperation, there exists considerable scope for mutually fruitful collaborative analysis of these mechanisms' workings, functions, and evolution across multiple species.

A second potentially fruitful collaboration is between agentbased or mathematical modeling of the conditions of evolution of tag-based cooperation and empirical research on the history of human evolution. Again, modeling work combining cultural inheritance of tags with genetic inheritance of behavioral strategies is in its infancy-most models hitherto presented serve at best as proof of product and "a basis for future studies that incorporate more realistic assumptions" (Ihara 2011:896). This work, however, appears to prioritize the quest for the most basic conditions under which tag-based cooperation can evolve. Models that are guided by realistic parameters of early human (African MSA onward) population structure, cognitive capabilities, social behaviors, tag inheritance mechanisms, and tag diversity and change could help generate and substantiate relevant predictions about the emergence and history of tag-based cooperation in humans.

Below, a series of concluding hypotheses summarize some core elements of an accent-tag-based account (i.e., accounts 1 and 2 above). If, specifically, accent facilitated the evolution of tag-based cooperation in humans and, ultimately, the psychological mechanisms that orient contingent cooperation along linguistic (and other group) lines, the following broad claims should be borne out by research on human behavior, cognition, evolution, and population dynamics, respectively:

1. Accent is a salient guide to social preferences in cooperation, trust, interaction, cultural learning, and norm acquisition (Giles 1977; Harwood and Giles 2005; Kinzler, Dupoux, and Spelke 2007); accent is privileged as a guide to social preferences over other comparable markers of population difference (Kinzler et al. 2009), controlling for degree of exposure, relevant cultural discourse, and so on.

2. Accent is a salient and privileged categorizer of individuals in attention and memory; accent guides social attitudes and biases in person perception, stereotyping, and evaluation (Gluszek and Dovidio 2010; Kristiansen 2001; Ryan 2008); "accent chameleons" who affect nonnative pronunciation and dialectal features evoke social attitudes and emotions in source and target accent groups (e.g., betrayal and ostracization or gratification and affiliation, Lakin et al. 2003; see also the literature on Foreign Accent Syndrome, e.g., Moen 2000); sensitivity to accent variation is calibrated by relevant experience, leading to finer tuned accent discrimination in one's native or long-term linguistic region than beyond it (Ikeno and Hansen 2007).

3. Changing populational and ecological pressures in human prehistory (e.g., increased community size, mobility, and dispersal into new environments; intermittent food scarcity) generated selective pressures that favored the emergence of tag-based mechanisms (as evidenced by, e.g., long-distance exchange of raw materials, and group and self-identification through artefact styles); existing diversity of vocalizations (as indexed, e.g., by anatomical prerequisites of sophisticated vocal imitation) permitted the emergence and maintenance of these mechanisms; rates of accent change over time meet the conditions required for the maintenance of cooperation in a tag-based model (Hales 2005).

4. Language variation and "speciation" is in part explainable in terms of social selection mechanisms (i.e., active selection or production of variants by individuals and groups; Irwin 1987); ecological risk, which predicts the size of social networks, should in turn predict the degree of language diversity across a population or geographical area (Nettle 1996); degrees of dispersal and migration (viscosity and mixing) in human populations are commensurate with parameters required for the emergence and maintenance of accent-tag cooperation (Hammond and Axelrod 2006a).

The proposals assessed and evolutionary scenarios speculated upon in this paper motivate a raft of questions and hypotheses that potentially span a wide range of relevant disciplines and methodologies. Until theoretical models and evidence are comprehensively and concomitantly examined, the specific evolutionary path, selective pressures and conditions, emergent properties, and long-term cycles of gene-culture coevolution in a tag-based model can only be speculated upon. As noted above, more research is required on whether and how the affordances and constraints of human culture (particularly linguistic signaling systems) satisfy the requirements of tag-based cooperative strategies. How much of human cooperation can potentially be explained by tag-based assortment, and how forms of cooperation might vary across increasing self-similarity distances, would be helpfully explored via mathematical models (e.g., following Antal et al. 2009). Proximate mechanisms and their ontogeny, in both humans and nonhumans, have only begun to be investigated in the recent developmental psychological and comparative literature. Whether accent is uniquely suited to the task of assorting cooperators with cooperation in a tag-based scenario, the ways in which accent tags are supplemented by other cues (such as appearance) and hard-to-fake communicative signals (including "common ground"), and the specific cultural conditions under which other forms of tag may 
rival or surpass the utility of accent tags in social interaction require further systematic consideration (Enfield 2006; Krupp, Debruine, and Barclay 2008). Nevertheless, existing findings within biology, psychology, anthropology, archaeology, and linguistics motivate concerted investment in further exploring these evidential gaps and in systematically reassessing the value of this deceptively simple idea.

\section{Acknowledgments}

I thank the anonymous reviewers of earlier versions of the manuscript for their many careful criticisms and insights, which served to improve the published paper considerably. For invaluable discussion, advice, and comments, I am especially grateful to Kathryn Cronin, Dan Dediu, Robin Dunbar, Nick Enfield, Matt Grove, Paul Harris, Daniel Haun, Kathryn Kinzler, Stephen Levinson, Daniel Nettle, Ryan Nichols, Harriet Over, Nadja Richter, Thom Scott-Phillips, and Harvey Whitehouse. They had many more questions than I had answers; all usual disclaimers apply.

\section{Comments}

\section{Quentin D. Atkinson}

Department of Psychology, University of Auckland, Private Bag 92019, Auckland, New Zealand (q.atkinson@auckland.ac.nz). 1 VI 12

\section{Accent as Social Sonar}

Game theoretic models show tag-based strategies are most advantageous when large group size and high dispersal rates limit the cooperation-enhancing effects of reputation, reciprocity, and kin proximity. Under these conditions, tags yield an advantage by providing new information about cooperative potential (Hammond and Axelrod 2006a).

One challenge for the argument Cohen puts forward is that early human social life was already primed for cooperation, with small, structured groups of individuals built around kinship and reciprocity. Later, symbolism and language created the possibility of group identity markers and sharing reputational information. Whether accent tags had a special role in facilitating the evolution of human cooperation depends on the existence of cooperative contexts in which accent provided new information. Cohen suggests that long-distance exchange networks evident in the African Middle Stone Age (MSA) may have provided this context (McBrearty and Brooks 2000). However, the archaeological record cannot tell us how these interactions played out and whether accent tags offered an advantage over the existing cooperative tool kit.

Data on social structure and cooperation in small-scale traditional societies may help us answer these questions. Re- cent work has shown that cooperation in hunter-gatherer social networks is clustered, such that variation is higher between neighboring groups than within groups, providing a meaningful level of intergroup variation for accent to track (Apicella et al. 2012). However, similar levels of cooperation only extend as far as two degrees of separation in the social network (Apicella et al. 2012). While this result is likely to be task specific, a preference at this range cannot be due to accent information. More work is needed into the nature and scale of different types of cooperative interactions in huntergatherer groups to establish where accent could play a role.

The scale of language variation is also important. We might expect the same cultural transmission processes that shape language diversity to also shape cooperative norms, such that accent variation reliably tracks variation in cooperation. To date, research into dialect variation has focused on languages of large-scale agricultural societies (Nerbonne 2010), which show distinct languages made up of many mutually intelligible regional dialects or accents. However, small-scale societies, of the sort that characterized much of our evolutionary history, show much greater variation at the level of languages. Vanuatu, for example, hosts more than 100 languages within a land area less than half the size of Belgium. When close neighbors are easily identified as an out-group because they speak a different language, there may be no advantage to cooperative preferences tied to subtle variation in accent.

Indeed, traditional societies tend to go to great lengths to advertise their group identity using markers such as clothing, tattoos, and body adornments. As Cohen points out, similar markers are thought to have existed in the MSA (Kuhn and Stiner $2007 a, 2007 b$ ). For accent to be a useful tag of cooperative potential, it would need to either provide new information about social distance (perhaps at a larger scale) or allow the identification of pretenders who fake group markers but cannot adjust their accent. Whether accent varies on an appropriate scale and whether pretenders could plausibly gain advantage were it not for accent remain unexplored.

Even if accent has not played a privileged role in our deep evolutionary past, as Cohen explains, it meets the requirements of a reliable, hard-to-fake tag, and many of us appear to use accent today to navigate our social worlds. It may be that accent has become a more salient group marker following the shift to agriculture and the emergence of hierarchical political structures comprising diffuse dialect continua and large population centers requiring regular cooperation with strangers.

More than just a tag, accent provides a uniquely finegrained measure of social distance that may have facilitated cooperation in large-scale agricultural societies. Individuals who share an accent may be more likely to have overlapping social networks. This can favor cooperation both by increasing concerns about the reputational consequences of noncooperation and by providing a proxy for the quality of available 
reputational information. ${ }^{1}$ Sensitivity to subtle variation in accent may therefore represent a unique social sense, a form of "social sonar," that allows us to navigate our social world in ways that have yet to be examined in game-theoretic simulations. A more recent role for accent fits with Cohen's account 3, that the tag-based system is not biologically specialized to use accent, but became useful in larger societies as cognitive systems for tracking socially relevant group markers were applied to the wealth of "free" information incorporated in accent.

\section{Dan Dediu and Mark Dingemanse ${ }^{2}$}

Language and Genetics, Max Planck Institute for Psycholinguistics, Wundtlaan 1, Nijmegen, NL-6525 XD, Netherlands (dan.dediu@ mpi.nl)/Language and Cognition, Max Planck Institute for Psycholinguistics, Wundtlaan 1, Nijmegen, NL-6525 XD, Netherlands. $26 \mathrm{~V} 12$

\section{More than Accent: Linguistic and Cultural Cues in the Emergence of Tag-Based Cooperation}

Emma Cohen presents a strong and well-informed case for the potential of culturally transmitted cues in tag-based cooperation and highlights "accent" as a prime candidate. Cohen's survey of the properties of viable tags introduces a conceptual apparatus that will shape future work in this domain, and her discussion of the way gradient tag traits enable the formation of flexible networks on different scales is a useful reminder of the dynamic and continuous (rather than stable and discrete) character of human sociality throughout evolutionary history. Here we highlight several issues that affect the generality and strength of the account, in particular the claim that accent might have a played a privileged role in the evolution of human cooperation.

Cohen seems to oscillate between different senses of "accent": in most places it seems to be a rather circumscribed notion of phonetic and prosodic variation, but in others it seems to include variation at any level of linguistic organization (including also the lexicon, grammar, and even pragmatics). This equivocation is indicative of a more general puzzle that ultimately remains unresolved in the paper: while "accent" (in the narrow sense) clearly has the potential to function as an effective tag, it is unclear why it should be privileged over other levels of linguistic variation that index social identities, and even over certain other culturally transmitted traits, something we return to below.

1. The "principle of information" (Pagel 2012) holds that we should adjust our willingness to cooperate to match the amount of information we have about a potential partner. Since reputational information flows along the same channels as language variation, accent could be an ideal proxy for quality of reputation information.

2. Both authors contributed equally to this comment, and author order is alphabetical.
Cohen's notion of (narrow) accent seems typical of large, agricultural, settled populations with long shared histories whose languages show significant internal variation. It is in such populations that many sociolinguistic studies have documented acute awareness of subtle gradient accentual differences. The main article cites this work in support of the case for "accent" in the evolution of human cooperation, but it is questionable whether it constitutes the most useful model. If "accent" is to have played an important role as a tag during human evolution, it must have been able to do its work in contexts more characteristic of preneolithic societies: small group sizes, high mobility, loose large-scale structures, fissionfusion dynamics, and possibly higher rates of local extinctions and recolonization (Eller, Hawks, and Relethford 2004; Ghiglieri 1987; Grove, Pearce, and Dunbar 2012; Hawks 2008). In many such contexts, subtle accentual variation would likely be overshadowed by more prominent cues. Moreover, if modern hunter-gatherers are a useful model, any tag would also have to cope with the possibility of high rates of linguistic divergence, multilingualism, and (linguistic) exogamy (Bowern 2010; Hill 1978). These phenomena are not addressed in the paper nor in modeling work in this domain, yet they all complicate a simple case for accent and emphasize the need for more empirical and modeling research and more attention to the social and demographic features of early humans and their ancestors.

What could be the alternative? We favor a more inclusive view, in which "accent" was but one possible ingredient of a complex dynamic set of tags. As not all cooperative contexts would have resulted in similar costs due to invasion by noncooperators, probably there was no single standard tag that kept cooperation going, but a flexible set of "tests" with different associated faking costs, the composition and relative importance of their components varying across place and time and depending on the context and content of particular interactions. While linguistic skills (including but not limited to "accent") may have been especially potent for the reasons mentioned in the paper, other plausible tags might include, for instance, body techniques (Mauss 1979), embodied cultural practices like walking style and posture (Bourdieu 1977; Youssouf et al. 1976), and permanent body adornments like tattooing and scarification (Joyce 2005), all of which are salient, hard to fake, and acquired relatively cost effectively in (early) socialization, and some of which may well be more ancient than linguistic cues. Even "biological" phenotypes such as facial features might play a role in this mix of possible tags as proxies for assessing genetic relatedness, not as simple greenbeard phenomena, but as one of many cues for a set of heuristics (Gigerenzer et al. 2000).

In sum, while we agree that "accent" makes a good tag (and possibly plays a role in tag-based cooperation in modern societies), we are unconvinced of the case for its privileged role in the evolution of human cooperation. Rather than looking for a single "magic bullet," we favor a model where accent was but one possible ingredient of a dynamic set of possible 
tags. The hunt for candidate tags is open: Cohen has made a case for accent, and we look forward to future research in which other cultural traits are assessed according to the conceptual framework developed in the paper. A complementary endeavor will be to provide a model for the integration and dynamic selection of the most appropriate tags for a given place, time, and interaction.

\section{Katherine D. Kinzler}

Department of Psychology, University of Chicago, 5848 South University Avenue, Chicago, IL 60637, U.S.A. (kinzler@uchicago .edu). 12 VI 12

Cohen's linguistic-tag-based account of human cooperation provides a discerning, carefully documented, and analytically forceful argument. Drawing on a large number of disciplines in the social sciences, she attempts the formidable task of describing the evolution of human cooperation and seeks explanatory power over many aspects of modern human sociality. As a psychologist, I am particularly intrigued by the interesting empirical predictions about human social behaviors that stem from Cohen's work. Some predictions are clear; furthermore, additional empirical inquiries could refine the theory and advance the synergistic dialogue between empiricists and theorists that Cohen so eloquently begins.

Cohen reviews experimental literature suggesting that, early in development, children attend to accent in guiding their early social preferences. Much recent research in developmental psychology (e.g., by Warneken, Tomasello, and colleagues) provides evidence that children are spontaneously helpful to others. Critically, interesting future experiments could probe whether children's earliest collaborative gestures are directed most reliably toward unknown others who exhibit a local accent.

Other interesting predictions can be made about the language-based social behavior of modern humans (both adults and children) based on Cohen's work. Cohen discusses the possibility of "accent chameleons," or those who convincingly assume a nonnative accent. If accent is interpreted as an honest signal of native-group membership that begets collaborative behavior, and a supposed native speaker speaks in a manner that betrays that categorization, he or she might be judged harshly. This idea could easily be tested in a laboratory setting. Potentially even more intriguingly, Cohen describes that a tag-based approach allows for some flexibility in the system. Depending on the ecological conditions at play, individuals could extend their range of accent tolerance to include a wider distinction if resources were plentiful or larger scale collaboration was advantageous, whereas they might shrink the range of accents that are accepted as "local" in situations of scarcity or when in competition with rival neighboring groups. Cohen argues that social selection mechanisms could thus be used to explain language variation and "spe- ciation" in modern times, where the degree of ecological risk across a geographic area could predict the degree of language diversity. I propose that such effects might be observed in a laboratory setting, too, which could shed additional light on the mechanisms that guide both linguistic perception and diversification. When placed in a simulated situation of either scarcity or plenty, listeners might differentially perceive linguistic boundaries in categorizing which accents would count as "in-group" versus "out-group."

Furthermore, the ideas presented in this paper could facilitate interesting experimental explorations concerning people's perception of accent as continuous versus dichotomous. One aspect of Cohen's model specifies that accents are comparable along a continuous dimension and that this linguistic continuity allows for measures of dialect difference. Indeed, research in sociolinguistics supports the idea that accents can change subtly and continuously over geographic spaces. Yet, Cohen also reviews evidence that individuals are better able to discriminate subtle differences in accents that are similar to their own accent, and that adults can even exhibit outgroup homogeneity effects whereby two foreign accents are not readily discriminated. To provide a further illustration of how these two ideas can intersect, although linguists acknowledge subtle variations in accent across geographic spaces in the United States, naïve perceivers often identify discrete speech regions such as "the South." As Cohen describes, accent variation can be used to maintain group boundaries, and decreased contact across social groups (which are also linguistically dissimilar) can result in decreasingly effective communication across group borders. Nonetheless, humans may also be inclined to perceive linguistic borders even in situations where the language change is gradual and continuous, and a discrete border may not in fact exist. Interesting open questions concern the nature of peoples' perception of accent as continuous versus dichotomous, and future research could continue to explore the psychological factors that contribute to exercises in linguistic line drawing.

Finally, I found myself contemplating two particular facets of the modern human linguistic landscape. The first is thatat least in modern times-a majority of human children are raised in bilingual or multilingual environments. The second is that modern accents indicate not only regional affiliation or geographic origins, but also status or prestige both within and across social groups that occupy geographically proximate spaces. Research in sociolinguistics (e.g., the work of Labov) demonstrates that variation in speech maps reliably to many aspects of social category membership, often among groups living in the same place at the same time. Further research might integrate proposals of accent as a tag-based strategy for cooperation with situations of multilingualism and with findings that demonstrate the pervasive instances of accent marking social status. 


\section{Robert Ladd and Lauren Hall-Lew}

Linguistics and English Language, University of Edinburgh, Dugald Stewart Building, 3 Charles Street, Edinburgh EH8 9AD, Scotland (bob@ling.ed.ac.uk).29 V 12

Cohen's idea strikes us as broadly plausible, and she is careful to allow the possibility of multiple versions of the detailed evolutionary sequence of events she sketches. We are not sure what would constitute clear counterevidence, but it would presumably be based on what is known about human prehistory, and this is beyond our expertise. Our comment therefore focuses on what is known about language variation, taking seriously Cohen's suggestion that research on the present can shed light on what must have happened in the past.

While her stance appears compatible with the complexity of patterns of accent production and perception that have been investigated in sociolinguistics, we think that she exaggerates the uniqueness of accent as an honest signal. For one thing, the availability of accent as an identity marker may depend on factors ranging from phonological structure to social structure: some groups rely more heavily on nonphonetic aspects of the linguistic system such as the lexicon (see, e.g., Smith and Johnson 1986 on elaborate clan-based variation in basic vocabulary in a small Australian aboriginal group). Furthermore, current research on the ethnography of speaking cautions against emphasizing the differences between accent and other cultural tags. Work in variationist sociolinguistics has demonstrated that the ability of a phonetic feature to signal group membership necessarily arises from its place in a broader symbolic system (e.g., Eckert [2000], who demonstrates close correlations between accent features and fine-grained nonlinguistic aspects of self-presentation). In other words, to the extent that accent features help signal group membership, they do so by virtue of their co-occurrence with a complex set of semiotic resources, including nonlinguistic cultural tags. Since the social meanings of accent and nonaccent features are coconstructed, and since this process occurs under intense, frequent, and ubiquitous social market pressures, nonaccent features might not be quite so "easy to fake" or "vulnerable to fraud" after all.

However, Cohen may underplay the significance of sociolinguistic variation in language acquisition. Her emphasis here is on how rapidly the ambient language begins to influence infant language development. She discusses evidence that by the end of the first year both the perception and production of vocalizations is significantly tuned to the language or languages that the infant hears and that some of these effects may already be detectable in newborns. Yet she also acknowledges that children may not be very sensitive to regional accents even by the time they start school. There is no contradiction here: it is just that her focus on the first year of life underestimates the cognitive investment that infants must make in mastering the basic mechanics of segmenting the stream of speech and acquiring a lexicon. More relevant evidence may come from the behavior of adults. A detailed study of variability in Tyneside English by Foulkes, Docherty, and Watt (2005) showed that adults speak differently to children as young as 2 depending on whether they are boys or girls. For example, when speaking to girls, adults were found to use the standard English pronunciation $[t]$ in words like water significantly more frequently than when they spoke to boys or other adults, when they used more common local variants (glottal stop, etc.) instead. The fact that adults unconsciously adjust the phonetic detail of the input they provide to linguistically immature children seems to us to provide an interesting line of evidence for the evolutionarily early emergence of language variation as a social marker, and in particular for the proposition that what accent provides is "honest information about childhood origins."

In closing, we note the monstrous multidisciplinary challenge posed by research into the origins of human behavior. Our field is linguistics; we are both phoneticians, with interests in cognitive and social aspects of language and language use. We were struck by how few of the works cited by Cohen we were familiar with, even those on accent variation, and we have suggested some ways in which her reading of sociolinguistics might be refined to serve her thesis better. The very fact that she has managed to synthesize the work she covers into a plausible explanatory framework deserves our admiration, but at the same time it serves as a reminder of how much more synthesizing will be needed as we progress.

\section{Arne Traulsen and Julián García}

Evolutionary Theory Group, Max-Planck-Institute for Evolutionary Biology, August-Thienemann-Str. 2, 24306, Plön, Germany (traulsen@evolbio.mpg.de). 18 V 12

\section{Chromodynamics of Accents?}

Our social preferences are unique, allowing us to pursue collective endeavors with strangers at remarkably large scales. A lot of research has been devoted to explain why we came to be that way. Models have played a critical role, exposing the consistency of possible accounts to mathematical rigor. However, evaluating competing hypotheses requires the thorough comparison of theoretical models with empirical evidence and experimental studies. Emma Cohen carefully builds such a bridge between mathematical models of tag-based cooperation and the literature on accents. In this note, we argue that tag reliability is a necessary but not sufficient condition for a tag to facilitate the evolution of cooperation.

The most basic models of the evolution of cooperation consider two types. Cooperators pay a cost to help others, and defectors avoid paying the cost while reaping benefits from cooperators. When everyone is equally likely to interact with everyone else, cooperation does not evolve. This changes if alike types are more likely to interact with each other. If helpers assort such that interactions among them are dispro- 
portionally frequent, then cooperation can evolve (Fletcher and Doebeli 2009). Within this framework, the role of models is to formalize and evaluate different mechanisms to produce such assortment. Tag-based cooperation is one such mechanism. If individuals cooperate with others that bear an arbitrary tag, cooperation is in principle possible. Help is preferentially channeled to those who share the tag, and thus cooperative interactions are assorted.

A way in which tag-based cooperation can work is if the tag and the cooperative behavior are tightly linked. This gives no room for faking the tag, and cooperation is stable (Haig 1996; Keller and Ross 1998). But if the tag and the cooperative behavior are only weakly linked, defectors can fake the tag, and cooperation breaks down. As a result, the plausibility of this mechanism is often judged on the existence of a "reliable" tag that is hard to fake. Cohen builds upon this and devotes a great deal of her argumentation toward convincing the readers that accents are reliable tags. However, the underlying reasoning arises from models that are inherently static and therefore ignore the dynamic nature of evolution.

In a model with mutations, any tag will be eventually faked. What makes a model work is that once a tag has been faked, a new signal must be established in order to exclude defectors. Whether tag-based cooperation can evolve therefore depends on who takes the lead in the race of faking and moving away from fakers. Two related and somehow contradictory characteristics determine this: as explained above, an appropriate tag needs to be hard to fake. But in addition, once the tag is forged, cooperators need the ability to swiftly establish another tag. What this implies is that tags need to be reliable, but they need to be inherently dynamic as well.

Emma Cohen makes a convincing case for accent reliability, but what about the capacity of accents to change once defectors have cracked them? This kind of dynamics is as important as tag reliability in theoretical models. Arguably, the dynamic nature of human language would seem to imply that accents are a good candidate for fluidity as well, but we believe that a systematic account on this issue, similar to the one presented for the case of reliability, would strengthen the case.

In addition, alternative models could incorporate the distinctive features of language and ancestral human interactions. For example, while many models of tag-based cooperation consider continuous tags in one or two dimensions (Antal et al. 2009; Riolo, Cohen, and Axelrod 2001) or even discrete tags (Jansen and Van Baalen 2006; Traulsen and Schuster 2003), accents may have multiple facets and are probably better described by multiple dimensions. This can be captured, for example, by set structured models in which every individual belongs to several sets, as described by Tarnita et al. (2009). Moreover, the large scale of human cooperation may not be described appropriately by pairwise interactions, dismissing more complex settings that could be particularly relevant in the case of humans (Gokhale and Traulsen 2010).

Theorists often note in passing that their tag-based models could be directly applicable to microbes, fads, or accents.
Often, such statements lack any empirical backup (e.g., Traulsen 2008). Cohen makes an important step toward a solid connection between such basic theoretical models and empirical accounts. While it may be sobering to see how few details of mathematical models turn out to have empirical relevance, it is important for modelers to understand on which level such a comparison is typically made.

The recipe for human sociality is likely to involve many ingredients, and tag-based cooperation based on accent may be one of them. In the end, it is unlikely that only one explanation will paint the complete picture of our social behavior, but evaluating the models in the light of empirical and experimental evidence is essential to determine which models matter, and how.

\section{Peter Trudgill}

Department of Foreign Languages, University of Agder, Post Box 422, 4604 Kristiansand, Norway (peter.trudgill@unifr.ch). 14 V 12

\section{On the Functionality of Linguistic Change}

Perhaps the greatest puzzle that faces linguistic scientists is the phenomenon of linguistic change. All languages change through time at all levels of language structure, but the biggest mysteries are associated with phonological and grammatical change. The changes that occur at these levels are cumulative, and the effects are drastic. No modern English speaker hearing a native speaker of Old English, from even as little as 1,000 years ago, would be able to understand them.

Why is this? Why do languages change? Linguists, when presented with this question-as they often are by lay audiences-tend to come up with answers of the type "languages are like that." But there are other questions too. Who changes a language? Clearly its speakers do, but they don't know they are doing it, and they certainly don't do it on purpose. And what is the point of language change?

There is a good case to be made for arguing that it is totally dysfunctional. As a language changes, it changes in different ways in different places. (The reasons for this are also not clear.) But it is this propensity that leads to the development of regional dialects and, in the fullness of time, to the development of these dialects into different and no longer mutually intelligible languages. As is well known, modern English, Dutch, German, Icelandic, and the continental Scandinavian languages are all descended from a single language now known to linguists as Germanic. Modern English, Dutch, and Swedish speakers are descended from Germanic speakers who were able understand one another, but we are no longer able to do so. This is truly rather startling. What could possibly be the advantage, evolutionary or otherwise, of an arrangement whereby the main means of communication between human beings has the inherent characteristic 
that it is subject to changes, the outcome of which is precisely the loss of the ability to communicate?

It is possible to argue that there may be some kind of advantage here, however. Languages are indeed a barrier to communication. But it can be suggested that the maintenance of a certain number of linguistic obstacles to communication might be advantageous. These barriers, although penetrable, ensure the survival of different language communities. And the separation of the world's population into different groups speaking different languages helps the growth and maintenance of cultural diversity, which in turn can lead to opportunities for the development of alternative modes of thought and of exploring possibilities for social, political, and technological development. A world where everyone spoke the same language might not only be rather dull but also a rather stagnant place.

But what about the earlier stages of linguistic divergence, where change has led simply to the development of different but still mutually intelligible dialects, with their associated accents? It is clear that the accent tags Cohen discusses are entirely due to language change. One of the biggest accent shibboleths in England has to do with the fact that words such as bath, laugh, pass are pronounced with a long ah vowel in the south of the country, while in the north they have the same short vowel as in bat, lap, pat. All English accents originally had the short vowel, but then in the 1700s, a changethe lengthening of the vowel before certain consonants-began in the London area and subsequently spread northward until it stopped in the English Midlands. ${ }^{3}$ At the point where it stopped, there was now an accent boundary-and a new accent tag, in Cohen's terms-which survives to this day.

We now see, from Cohen's highly insightful paper, that this kind of development may not be dysfunctional either, from an evolutionary perspective. Accents are acquired very early on in the human child's development. They are the result of infants' extraordinary ability to acquire precisely the pronunciation habits of those around them. This requires synchronized micromillimeter and microsecond-accurate control of the lips, tongue, velum, jaws, and vocal cords in order to produce precisely the right sounds, which is why it is enormously difficult for adults to acquire a second language/ dialect/accent perfectly. "Faking" is therefore extremely difficult, in a way that it is not with vocabulary-anybody can readily acquire a new word, and word usage can be subjected to conscious control. It is no surprise either that it is phonology that has acquired the tag function, to a much greater extent than grammar. Grammatical features may occur only very infrequently, while the vowels and consonants that accents consist of cannot be avoided, and occur with very high frequency indeed.

3. Why it stopped exactly where it did is also slightly mysterious, although dialectologists have been able to isolate a number of explanatory factors (see Chambers and Trudgill 1998).

\section{Reply}

I thank my commentators for the careful reflection so evident in their responses. Commentaries represent the range of disciplinary perspectives drawn upon in my paper, offering important new insights and suggestions for developing and testing accounts of tag-based cooperation in humans.

The aim motivating the paper was twofold: (1) to consider the relevance for human cooperation of recent work in theoretical biology on the wide range of conditions under which tag- and similarity-based cooperation could evolve and (2) to assess the empirical plausibility of recent claims concerning social assortment via accent as part of a phylogenetically specialized cognitive system. The primary criticism hitherto leveled at tagging accounts is that no socially acquired trait is sufficiently honest to guide cooperation in humans. I argue that theoretical and empirical developments in relevant disciplines warrant a reappraisal of the case.

It is notable that that discussion of tag honesty is largely absent from commentaries (barring Trudgill's supportive final paragraph and Kinzler's interest in the ideas on accent chameleons). That a wider range of ideas, questions, and future research directions have emerged from the discussion thus far is encouraging, irrespective of whether the new appeal and continuing debate will ultimately lead us again to dismiss the case.

Despite broad interdisciplinary representation, there is considerable agreement on the principal theoretical and empirical challenges that a plausible account must address. All commentators expand the scope of debate to query the viability of accent as a useful assortment mechanism within relevant conditions of human evolution. Atkinson, Dediu and Dingemanse, Ladd and Hall-Lew, and Traulsen and Garcia, in particular, stress that the viability of a tag-based system hinges on tag variation across space and tag fluidity over time, and that this should be assessed in relation to population size and structure.

A number of comments prompt me to begin with some clarifications. First, the primary focus of the paper, and the tagging account, is accent (phonetic and prosodic patterns, or pronunciation), not vocabulary and grammar. Second, I do not argue that accent is a uniquely honest or suitable tag; rather, I selected accent as a candidate tag on which to focus the case. Third, it is not the case that tagging (via accent or some other trait) helps explain the whole of human cooperation-tag-based cooperation would have emerged in the context of a preexisting set of mechanisms, constraints, and opportunities for cooperation on a smaller scale. The specific evolutionary and contextual conditions under which tagging became useful, how much and what kinds of cooperation it guides, how it interacts with other mechanisms, and how, in turn, it may have provided the foundation for new mechanisms for cooperation are open questions about which I begin 
to speculate in the final section. Fourth, the case does not assume a priori that tags are group-level markers of identity (e.g., of clan or childhood origins). They are observable phenotypic traits that guide assortment among individuals on the basis of interindividual similarity/difference.

Finally, in light of recent claims about the specialness of accent in social preferences and cognitive evolution, the paper assesses accent tagging primarily as a phylogenetically specialized system. In the final section, however, I sketch three accounts that concern the evolutionary sources from which the assortment of cooperators via accent tags might have emerged. Account 1 entails natural selection on the accent tagging system wholesale. Account 2 entails natural selection on elements of the tagging system independently, which in turn operate conjointly under certain population-structure conditions. Account 3 entails ontogenetic specialization of a general self-similarity mechanism to tags that relevantly differentiate individuals within a particular environment (potentially including, but not limited to, accent). By all accounts, those individuals who exploited tags in assortment had a fitness advantage over those who did not. However, accounts 1 and 2, which entail selection for the system or its constituent parts, demand a different set of conditions/pressures and adaptations from account 3 , in which an evolved tendency to favor similar others (that may have built on existing mechanisms for kin selection) is tuned through experience to available, relevant, and reliable social cues.

\section{Accent Variation in Space}

Atkinson, and Dediu and Dingemanse query whether accents varied sufficiently (relevantly) across early human groups and populations to guide fine-grained cooperative strategies. Although the broad scale and scope of accent variation in large monolingual societies are undoubtedly historically very recent, relevant ethnography also yields ample evidence of the widespread importance of within-language variation for sociality across small-scale societies. Sankoff (1980:112), for example, observes that "virtually all over Papua New Guinea, people pay very careful attention to small linguistic differences in differentiating themselves from their neighbours" and cites evidence that individuals attend specifically to fine-grained phonological differences and even mimic the speech patterns of others in linguistic play. Similarly, among the Bugisu of eastern Uganda, according to Heald (1989), within-language variation provided the main criteria for the perception of cultural difference, including variations in very minute distinctions between the speech of subclans. A range of ethnographic literature in the Human Relations Area Files consistently characterizes linguistic variation over large populations and territories of the San in terms of within-language variation. It is interesting for the current account that the dialect group was sufficiently large that no single family had contact with most members and consequently there was no strong sense of identity for the group as a whole. These are precisely the conditions in which tags, or similarity-based assessments, could become useful guides to cooperation. Indeed, Wiessner (1977) identified a wide range of cooperative interactions, including marriage, that preferentially took place within San dialect groups.

Cherry-picking examples is not particularly helpful, however. We need a systematic and quantitative survey of the literature that allows for hypothesis testing about the relationships between population demographics and accent variation. Quantitative analysis on specifically phonological variation over large populations is highly laborious work, however, and presents many methodological challenges of representation (of speakers and linguistic samples recorded) and comparison (of available speaker-language samples).

Where relevant data exist, methodological developments in dialectometry (referenced in the main article) open fascinating new prospects for increasingly accurate measurement and classification of, specifically, accent variation. Consistent with Kinzler's remarks, these methods highlight the continuities among the speech variants of neighboring groups that often make language versus dialectical versus accent distinctions difficult for the linguist to establish on the ground. Hockett (1958) reports that speakers in parts of West Africa characterize degrees of linguistic difference according to the period it takes to establish easy communication between related forms of speech-for example, the speech variant of an adjacent village may be described as a "2-day" dialect and that of a more distant village as a "5-day" dialect. This method perhaps hints at a potentially more relevant, sociolinguistically informed measure for our purposes than the awkward divides that often characterize traditional linguistic classifications.

The methods by which we measure and carve up variation have important implications for characterizations of monolingualism and multilingualism. I appreciate Kinzler's and Dediu and Dingemanse's remarks on the potential challenge of multilingualism for the account (and Ladd and Hall-Lew's related insights on input variations in language acquisition). A systematic treatment of this complex issue could potentially begin with an attempt to separate out the properties and functions of accent from those of language. There is a common tendency to view accent as taxonomically subordinate to language, but prosodic patterns and phonetic repertoires can extend across neighboring and related languages such that unintelligible speech variants can sound familiar or local. Even in majority monolingual societies, we find cases of monoaccented multilingualism (e.g., in Northern Irish English and Gaelic), in which two mutually unintelligible languages are spoken with the same or recognizably similar regional accent. In principle, multilingualism need not entail mastery of diverse phonetic repertoires, particularly among neighboring communities. It could even be the case that, even where language diversity is high, language and accent distinctions play different functions, with language boundaries marking one sphere of cooperative interaction, and accents marking a 
broader sphere over the "middle distance" (Kuhn and Stiner 2007b).

\section{Accent Variation over Time}

Among the most interesting findings from recent tag models are the insights that tag-based cooperation can evolve in a population if tags mutate faster than strategies (Hales 2005), and it can reemerge after free-rider invasion if new populations of cooperators regroup around new tags (see Traulsen and Garcia's commentary). Although debate within anthropology has focused on whether humans possess tags that are sufficiently hard to fake, tag honesty is a limiting but insufficient condition for the evolution of tag-based assortment. Traulsen and Garcia clearly explain why honesty and change must be considered conjointly in models in which tags and strategies are weakly linked, and they are right to request a systematic treatment on accent fluidity over time. Further models that are informed by empirical principles of linguistic change, such as those Trudgill describes in his commentary, would be informative here. Trudgill identifies linguistic change as "the greatest puzzle that faces linguistic scientists." Nevertheless, general principles governing patterns of drift, splitting, merging, shifting, and so on could provide a helpful foundation on which to model and measure threshold rates of accent fluidity for tag-based cooperation to emerge and be sustained. We might hypothesize, for example, that the splintering events (e.g., free-rider invasions) that prompt regrouping around new tags would motivate rapid linguistic change, but that the countervailing constraints on tag change maintain a relatively slow dynamic otherwise. There is some empirical evidence from evolutionary linguistics to support this hypothesis. In a phylogenetic analysis of one-third of the world': languages, representing thousands of years of language evolution, Atkinson et al. (2008) revealed a pattern of punctu. ational bursts: rapid early language change is followed by a period of gradual and slower divergence.

Finally, I agree with Atkinson (as well as Dediu and Dingemanse, and Ladd and Hall-Lew) that analysis of propertie: of present-day hunter-gatherer social networks may help answer questions about how dynamic linguistic variation mapped onto population structure in ancestral populations. In the cited study with Hadza hunter-gatherers (Apicella et al. 2012), assortment among similarly cooperative cooperators in such a small group $(N=205)$ was probably achieved vi; mechanisms other than tagging — specifically, social proximity, genetic proximity, and camp comembership.

Nevertheless, the significant effects of homophily- "the tendency of similar people to form ties" (Apicella et al. 2012: 497) —on Hadza social assortment suggests the importance of general self-similarity mechanisms in line with account ? (see also Krupp, Debruine, and Barclay 2008; Lusseau and Newman 2004; McPherson, Smith-Lovin, and Cook 2001, Wey and Blumstein 2010). Tag and similarity-based assortment mechanisms are agreed to be relevant and useful wher individuals' knowledge of kinship, opportunities for reciprocity, or availability of reliable reputational information on potential interaction partners are limited, but the roots of these mechanisms may be in older similarity-preference mechanisms guiding assortative interactions in smaller scale societies. These mechanisms may exploit accent similarities but may also flexibly tune into alternative sources of relevant variation in the environment. For example, in a modern population characterized by accent homogeneity and racial heterogeneity, self-similarity mechanisms may display sensitivity to racial differences in social assortment. Moreover, they may attend to more than one tag or similarity measure (Dediu and Dingemanse; Ladd and Hall-Lew) and to additional traits that similarity reliably references (Atkinson). Tag-based systems based on aggregate tags could ameliorate honesty/fluidity constraints required within a single-tag model.

Space constraints do not permit adequate discussion of these and alternative or additional possibilities (e.g., those considering harming behaviors and complementarity in between-tag interactions) and of the full range of suggestions my distinguished commentators have made. Nevertheless, that the account's potential and complexities are only beginning to be understood will, I hope, serve to inspire and cement the importance of continued debate, collaboration, and research.

—Emma Cohen

\section{References Cited}

Aiello, L., and R. Dunbar. 1993. Neocortex size, group size, and the evolution of language. Current Anthropology 34:184-193.

Alford, R. L., and J. B. Strother. 1990. Attitudes of native and nonnative speakers toward selected regional accents of US English. TESOL Quarterly 24:479-495.

Antal, T., H. Ohtsuki, J. Wakeley, P. D. Taylor, and M. A. Nowak. 2009. Evolution of cooperation by phenotypic similarity. Proceedings of the $\mathrm{Na}$ tional Academy of Sciences of the USA 106:8597-8600.

$\rightarrow$ Apicella, C. L., F. W. Marlowe, J. H. Fowler, and N. A. Christakis. 2012. Social networks and cooperation in hunter-gatherers. Nature 481:497-501. [QDA]

Atkinson, Q. D., A. Meade, C. Venditti, S. J. Greenhill, and M. Pagel. 2008. Languages evolve in punctuational bursts. Science 319:588.

$\rightarrow$ Axelrod, R., R. A. Hammond, and A. Grafen. 2004. Altruism via kin-selection strategies that rely on arbitrary tags with which they coevolve. Evolution 58:1833-1838.

Baker, M. C. 2002. The atoms of language. Oxford: Oxford University Press.

Bar-Haim, Y., T. Ziv, D. Lamy, and R. M. Hodes. 2006. Nature and nurture in own-race face processing. Psychological Science 17(2):159-163.

Baxter, G. J., R. A. Blythe, W. Croft, and A. J. McKane. 2009. Modeling language change: an evaluation of Trudgill's theory of the emergence of New Zealand English. Language Variation and Change 21(2):257-296.

$\rightarrow$ Berg, K. S., S. Delgado, K. A. Cortopassi, S. R. Beissinger, and J. W. Bradbury. 2012. Vertical transmission of learned signatures in a wild parrot. Proceedings of the Royal Society B: Biological Sciences 279(1728):585-591.

Billig, M., and H. Tajfel. 1973. Social categorization and similarity in intergroup behaviour. European Journal of Social Psychology 3:27-52.

Blommaert, Jan. 2009. Language, asylum, and the national order. Current Anthropology 50(4):415-441.

$\rightarrow$ Bocquet-Appel, J. P., and P. Y. Demars. 2000. Population kinetics in the Upper Palaeolithic in western Europe. Journal of Archaeological Science 27:551570.

Bocquet-Appel, J. P., P. Y. Demars, L. Noiret, and D. Dobrowsky. 2005. Es- 
timates of Upper Palaeolithic meta-population size in Europe from archaeological data. Journal of Archaeological Science 32:1656-1668.

$\rightarrow$ Boughman, J. W., and G. S. Wilkinson. 1998. Greater spear-nosed bats discriminate group mates by vocalizations. Animal Behaviour 55:1717-1732.

Bourdieu, Pierre. 1977. Outline of a theory of practice. R. Nice, trans. Cambridge: Cambridge University Press. [DD, MD]

$\rightarrow$ Bowern, Claire. 2010. Correlates of language change in hunter-gatherer and other "small" languages. Language and Linguistics Compass 4:665-679. [DD, $\mathrm{MD}]$

$\rightarrow$ Bowles, S., and H. Gintis. 2004. The evolution of strong reciprocity: cooperation in heterogeneous populations. Theoretical Population Biology 65 17-28.

$\rightarrow$ Boyd, R., H. Gintis, S. Bowles, and P. J. Richerson. 2003. The evolution of altruistic punishment. Proceedings of the National Academy of Sciences of the USA 100:3531-3535.

Boyd, R., and P. J. Richerson. 1985. Culture and the evolutionary process. Chicago: University of Chicago Press.

. 2005. The origin and evolution of cultures. New York: Oxford University Press.

$\rightarrow$. 2009. Culture and the evolution of human cooperation. Philosophical Transactions of the Royal Society B 364:3281-3288.

$\rightarrow$ Bresnahan, M. J., R. Ohashi, R. Nebashi, W. Y. Liu, and S. M. Shearman. 2002. Attitudinal and affective response toward accented English. Language and Communication 22:171-185.

$\rightarrow$ Brinck, I., and P. Gärdenfors. 2003. Co-operation and communication in apes and humans. Mind and Language 18:484-501.

Burnham, T. C., and D. D. P. Johnson. 2005. The biological and evolutionary logic of human cooperation. Analyse \& Kritik 27:113-135.

$\rightarrow$ Castro, L., and M. A. Toro. 2007. Mutual benefit cooperation and ethnic cultural diversity. Theoretical Population Biology 71(3):392-399.

$\rightarrow$ Cavalli-Sforza, L. L., and B. Hewlett. 1982. Exploration and mating range in African Pygmies. Annals of Human Genetics 46:257-270.

Chambers, J. K., and P. Trudgill. 1998. Dialectology. Cambridge: Cambridge University Press. [PT]

Chambers, J. K., P. Trudgill, and N. Schilling-Estes. 2002. The handbook of language variation and change. Malden, MA: Blackwell.

$\rightarrow$ Chudek, M., and J. Henrich. 2011. Culture-gene coevolution, norm-psychology and the emergence of human prosociality. Trends in Cognitive Sciences 15:218-226.

$\rightarrow$ Clopper, C. G. 2008. Auditory free classification: methods and analysis. Behavior Research Methods 40:575-581.

$\rightarrow$ Clopper, C. G., and D. B. Pisoni. 2006. Effects of region of origin and geo graphic mobility on perceptual dialect categorization. Language Variation and Change 18:193-221.

$\rightarrow$. 2007. Free classification of regional dialects of American English. Journal of Phonetics 35:421-438.

$\rightarrow$ Colman, A., L. Browning, and B. D. Pulford. 2012. Spontaneous similarit, discrimination in the evolution of cooperation. Journal of Theoretical Biology 299:162-171.

$\rightarrow$ Cross, I. 2009. Communicative development: neonate crying reflects patterns of native-language speech. Current Biology 19:R1078-R1079.

$\rightarrow$ Dailey, R. M., H. Giles, and L. L. Jansma. 2005. Language attitudes in an Anglo-Hispanic context: the role of the linguistic landscape. Language and Communication 25:27-38.

Dawkins, R. 1976. The selfish gene. New York: Oxford University Press. . 1982. The extended phenotype. Oxford: Oxford University Press.

$\rightarrow$ Day, R. R. 1980. The development of linguistic attitudes and preferences TESOL Quarterly 14(1):27-37.

de Boysson-Bardies, B. 1993. Ontogeny of language-specific syllabic produc tions. In Developmental neurocognition: speech and face processing in the firs year of life. B. de Boysson-Bardies, S. de Schonen, P. Jusczyk, P. McNeilage, and J. Morton, eds. Pp. 353-363. Dordrecht: Kluwer.

$\rightarrow$ Dediu, D. 2011. Are languages really independent from genes? if not, what would a genetic bias affecting language diversity look like? Human Biolog, 83:279-296.

$\rightarrow$ Dixon, J., B. Mahoney, and R. Cocks. 2002. Accents of guilt? effects of regional accent, race, and crime on attributions of guilt. Journal of Language and Social Psychology 21:162-168.

Dunbar, R. 1996. Grooming, gossip, and the evolution of language. London: Faber \& Faber.

. 1999. Culture, honesty and the free rider problem. In The evolution of culture. R. Dunbar, C. Knight, and C. Power, eds. Pp 194-213. Edinburgh Edinburgh University Press.
2008. Mind the gap; or why humans are not just great apes. Proceedings of the British Academy 154:403-423.

. 2009. Why only humans have language. In The prehistory of language. R. Botha and C. Knight, eds. Pp 12-35. Oxford: Oxford University Press. Eckert, Penelope. 2000. Linguistic variation as social practice. Malden, MA: Blackwell. [DRL, LH-L]

Edwards, J. 2009. Language and identity. Cambridge: Cambridge University Press.

Eimas, P. D., E. R. Siqueland, P. Jusczyk, and J. Vigorito. 1971. Speech perception in infants. Science 171:303-306.

Eller, E., J. Hawks, and J. H. Relethford. 2004. Local extinction and recolonization, species effective population size, and modern human origins. $\mathrm{Hu}$ man Biology 76:689-709. [DD, MD]

Enfield, N. J. 2006. Social consequences of common ground. In Roots of human sociality: culture, cognition and interaction. N. J. Enfield and S. C. Levinson, eds. Pp. 399-430. Oxford: Berg.

Engeszer, R. E., M. J. Ryan, and D. M. Parichy. 2004. Learned social preference in zebrafish. Current Biology 14:881-884.

$\rightarrow$ Eshel, I., and L. L. Cavalli-Sforza. 1982. Assortment of encounters and evolution of cooperativeness. Proceedings of the National Academy of Sciences of the USA 79:1331-1335.

Feekes, F. 1977. Colony-specific song in Cacicus cela (Icteridae, Aves): the password hypothesis. Ardea 65:197-202.

$\rightarrow$ Fehr, E., and U. Fischbacher. 2003. The nature of human altruism. Nature 425:785-791.

Feldman, R. E. 1968. Response to compatriot and foreigner who seek assistance. Journal of Personality and Social Psychology 10:202-214.

Ferguson, C. A., L. Menn, and C. Stoel-Gammon, eds. 1992. Phonological development: models, research, implications. Timonium, MD: York.

Fisher, R. A. 1930. The genetical theory of natural selection. Oxford: Clarendon.

$\rightarrow$ Fitch, T. 2000. The evolution of speech: a comparative review. Trends in Cognitive Sciences 4:258-267.

$\rightarrow$ Fletcher, J. A., and M. Doebeli. 2009. A simple and general explanation for the evolution of altruism. Proceedings of the Royal Society B 276:13-19.

$\rightarrow$ Floccia, C., F. Butler, and J. Goslin. 2009. Categorization of regional and foreign accent in 5- to 7-year-old British children. International Journal of Behavioral Development 33:366-375.

Foley, R. A. 2004. The evolutionary ecology of linguistic diversity in human populations. In Traces of ancestry: studies in honour of Colin Renfrew. M. Jones, ed. Pp. 61-71. Oakville, CT: David Brown Book Company.

Foley, R. A., and C. Gamble. 2009. The ecology of social transitions in human evolution. Philosophical Transactions of the Royal Society B 364:3267-3279.

Ford, J. K. B. 1991. Vocal traditions among resident killer whales (Orcinus orca) in coastal waters of British Columbia. Canadian Journal of Zoology 69:1454-1483.

Foulkes, Paul, Gerard Docherty, and Dominic Watt. 2005. Phonological variation in child-directed speech. Language 81:177-206. [DRL, LH-L]

Fuertes, J. N., J. C. Potere, and K. Y. Ramirez. 2002. Effects of speech accents on interpersonal evaluations: implications for counseling practice and research. Cultural Diversity and Ethnic Minority Psychology 8:346-356.

Gamble, C. 1983. Culture and society in the Upper Paleolithic of Europe. In Hunter-gatherer economy in pre-history. G. N. Bailey, ed. Pp. 201-211. Cambridge: Cambridge University Press.

$\rightarrow \ldots$. 1998. Palaeolithic society and the release from proximity: a network approach to intimate relations. World Archaeology 29:426-449.

$\rightarrow$ Gardner, A., and A. Grafen. 2009. Capturing the superorganism: a formal theory of group adaptation. Journal of Evolutionary Biology 22:659-671.

Gardner, A., and S. A. West. 2010. Greenbeards. Evolution 64:25-38.

Ghiglieri, Michael P. 1987. Sociobiology of the great apes and the hominid ancestor. Journal of Human Evolution 16:319-357. [DD, MD]

Gigerenzer, Gerd, Peter M. Todd, and the ABC Research Group. 2000. Simple heuristics that make us smart. Oxford: Oxford University Press. [DD, MD]

Giles, H. 1973. Communicative effectiveness as a function of accented speech. Speech Monographs 40:330-331. Press.

$\rightarrow$ Gintis, H. 2000. Strong reciprocity and human sociality. Journal of Theoretical Biology 206:169-179.

$\rightarrow$ Gintis, H., S. Bowles, R. Boyd, and E. Fehr. 2003. Explaining altruistic behavior in humans. Evolution and Human Behavior 24:153-172.

Girard, F., C. Floccia, and J. Goslin. 2008. Perception and awareness of accents in young children. British Journal of Developmental Psychology 26:409-433. 
$\rightarrow$ Gluszek, A., and J. F. Dovidio. 2010. The way they speak: a social psychological perspective on the stigma of nonnative accents in communication. Personality and Social Psychology Review 14:214-237.

$\rightarrow$ Gokhale, C. S., and A. Traulsen. 2010. Evolutionary games in the multiverse. Proceedings of the National Academy of Sciences of the USA 107:5500-5504. [AT, JG]

$\rightarrow$ Goodfellow, D. J., and P. J. B. Slater. 1986. A model of bird song dialects. Animal Behaviour 34:1579-1580.

$\rightarrow$ Grafen, A. 2006. Optimisation of inclusive fitness. Journal of Theoretical Biology 238:541-563.

Grove, M. 2010a. The archaeology of group size. Proceedings of the British Academy 158:391-411.

$\rightarrow-2010 b$. Logistical mobility reduces subsistence risk in hunting economies. Journal of Archaeological Science 37:1913-1921.

$\rightarrow$ Grove, M., E. Pearce, and R. I. M. Dunbar. 2012. Fission-fusion and th evolution of hominin social systems. Journal of Human Evolution 62:191200. [DD, MD]

$\rightarrow$ Hadzibeganovic, T., D. Stauffer, and C. Schulze. 2008. Boundary effects in a three-state modified voter model for languages. Physica A 387:3242-3252.

$\rightarrow$ Haig, D. 1996. Gestational drive and the green-bearded placenta. Proceedings of the National Academy of Sciences of the USA 93:6547-6551. [AT, JG]

Hales, D. 2005. Change your tags fast! a necessary condition for cooperation MultiAgent and MultiAgentBased Simulation LNAI 3415:89-98.

$\rightarrow$ Hamilton, W. D. 1964. The genetical evolution of social behaviour. Journa $\rightarrow$ of Theoretical Biology 7:1-52.

. 1975. Innate social aptitudes of man: an approach from evolutionar genetics. In Biosocial anthropology. R. Fox, ed. Pp.133-155. New York: Wiley.

$\rightarrow$ Hammond, R. A., and R. Axelrod. 2006a. Evolution of contingent altruism when cooperation is expensive. Theoretical Population Biology 69:333-338

$\rightarrow-2006 b$. The evolution of ethnocentrism. Journal of Conflict Resolution 50:926-936.

Harwood, J., and H. Giles, eds. 2005. Intergroup communication: multiple perspectives. New York: Peter Lang.

$\rightarrow$ Hausberger, M., E. Bigot, and P. Clergeau. 2008. Dialect use in large assemblies: a study in European starling Sturnus vulgaris roosts. Journal of Avian Biology 39:672-682.

Hawks, J. 2008. From genes to numbers: effective population sizes in human evolution. In Recent advances in paleodemography. J.-P. Bocquet-Appel, ed Pp. 9-30. Dordrecht: Springer. [DD, MD]

Heald, S. 1989. Controlling anger: the sociology of Gisu violence. Manchester: Manchester University Press.

$\rightarrow$ Heeringa, W., and J. Nerbonne. 2001. Dialect areas and dialect continua. Language Variation and Change 13:375-400.

$\rightarrow$ Henrich, J. 2004. Cultural group selection, coevolutionary processes and largescale cooperation. Journal of Economic Behavior and Organization 53:3-35. and Reply to commentaries, pp. 127-143.

Henrich, J., and N. Henrich. 2007. Why humans cooperate: a cultural and evolutionary explanation. New York: Oxford University Press.

$\rightarrow$ Herbinger, I., S. Pepworth, C. Boesch, and K. Zuberbüler. 2009. Vocal, gestural and locomotor responses of wild chimpanzees to familiar and unfamiliar intruders: a playback study. Animal Behaviour 78:1389-1396.

$\rightarrow$ Hewlett, B., J. M. H. Vandekoppel, and L. L. Cavalli-Sforza. 1982. Exploration ranges of Aka Pygmies of the Central African Republic. Man 17:418-430.

Heyes, C. Forthcoming. What can imitation do for cooperation? In Signalling, commitment and emotion. Cambridge, MA: MIT Press.

$\rightarrow$ Hill, Jane H. 1978. Language contact systems and human adaptations. Journal of Anthropological Research 34:1-26. [DD, MD]

$\rightarrow$ Hill, K. 2002. Cooperative food acquisition by Ache foragers. Human Nature 13:105-128.

Hockett, C. F. 1958. A course in modern linguistics. New York: Macmillan.

Hollien, H. 2002. Forensic voice identification. San Diego, CA: Academic Press.

$\rightarrow$ Hopp, S. L., P. Jablonski, and J. L. Brown. 2001. Recognition of group membership by voice in Mexican jays, Aphelocoma ultramarina. Animal Behaviour 62:297-303.

Howley, E., and J. Duggan. 2010. The effects of evolved sociability in a com mons dilemma. Adaptive and Learning Agents, LNAI 5924:38-48.

$\rightarrow$ Ihara, Y. 2011. Evolution of culture-dependent discriminate sociality: a gene culture coevolutionary model. Philosophical Transactions of the Royal Society B: Biological Sciences 266:889-900.

$\rightarrow$ Ikeno, A., and J. H. L. Hansen. 2007. The effect of listener accent backgrounc on accent perception and comprehension. EURASIP Journal on Audio, Speech, and Music Processing 2007:1-9.

Irwin, C. J. 1987. A study in the evolution of ethnocentrism. In The sociobiolog, of ethnocentrism. V. Reynolds, V. S. E. Falger, and I. Vine, eds. Pp. 131156. London: Croom Helm.

Janik, V. M., L. S. Sayigh, and R. S. Wells. 2006. Signature whistle shape conveys identity information to bottlenose dolphins. Proceedings of the $\mathrm{Na}$ tional Academy of Sciences of the USA 103:8293-8297.

$\rightarrow$ Jansen, V. A. A., and M. van Baalen. 2006. Altruism through beard chromodynamics. Nature 440:663-666.

Jessen, M. 2007. Speaker classification in forensic phonetics and acoustics. Speaker Classification I, LNAI 4343:180-204.

Johnson, A. W., and T. K. Earle. 2000. The evolution of human societies: from foraging group to agrarian state. Stanford, CA: Stanford University Press.

Joyce, Rosemary A. 2005. Archaeology of the body. Annual Review of Anthropology 34(1):139-158, doi:10.1146/annurev.anthro.33.070203.143729. [DD, MD]

Kaplan, H., K. Hill, R. V. Cadeliña, B. Hayden, D. C. Hyndman, R. J. Preston, E. A. Smith, D. E. Stuart, and D. R. Yesner. 1985. Food sharing among Ache foragers: tests of explanatory hypotheses. Current Anthropology 26: 223-246.

Kaplan, H., K. Hill, and A. M. Hurtado. 1990. Risk, foraging, and food sharing among the Ache. In Risk and uncertainty in tribal and peasant economies. E. Cashdan, ed. Pp. 107-143. Boulder, CO: Westview.

Keller, L., and K. G. Ross. 1998. Selfish genes: a green beard in the red fire ant. Nature 394:573-575. [AT, JG]

Kinzler, K. D., K. H. Corriveau, and P. L. Harris. 2011. Children's selective trust in native-accented speakers. Developmental Science 14:106-111.

Kinzler, K. D., E. Dupoux, and E. S. Spelke. 2007. The native language of social cognition. Proceedings of the National Academy of Sciences of the USA 104:12577-12580.

Kinzler, K. D., K. Shutts, J. DeJesus, and E. S. Spelke. 2009. Accent trumps race in guiding children's social preferences. Social Cognition 27:623-634.

$\rightarrow$ Kinzler, K. D., K. Shutts, and E. S. Spelke. 2012. Language-based social preferences among children in South Africa. Language Learning and Development 8(3):215-232.

Kristiansen, G. 2001. Social and linguistic stereotyping: a cognitive approach to accents. Estudios Ingleses de la Universidad Complutense 9:129-146.

$\rightarrow$ Krupp, D. B., L. M. Debruine, and P. Barclay. 2008. A cue of kinship promotes cooperation for the public good. Evolution and Human Behavior 29:49-55.

Kuhl, P. K., E. Stevens, A. Hayashi, T. Deguchi, S. Kiritani, and P. Iverson. 2006. Infants show a facilitation effect for native language phonetic perception between 6 and 12 months. Developmental Science 9:F13-F21.

Kuhn, S. L., and M. Stiner. $2007 a$. Body ornamentation as information technology: towards an understanding of the significance of early beads. In Rethinking the human revolution. P. Mellars, K. Boyle, O. Bar-Yosef, and C. Stringer, eds. Pp. 45-54. Cambridge: MacDonald Institute of Archaeology.

. 2007b. Paleolithic ornaments: implications for cognition, demography and identity. Diogenes 54:40-48.

Kurzban, R., J. Tooby, and L. Cosmides. 2001. Can race be erased? coalitional computation and social categorization. Proceedings of the National Academy of Sciences of the USA 98:15387-15392.

Ladd, D., D. Dediu, and A. Kinsella. 2008. Languages and genes: reflections on biolinguistics and the nature-nurture question. Biolinguistics 2:114-126.

Lakin, J. L., V. E. Jefferis, C. M. Cheng, and T. L. Chartrand. 2003. The chameleon effect as social glue: evidence for the evolutionary significance of nonconscious mimicry. Journal of Nonverbal Behavior 27:145-162.

Layton, R., and S. O'Hara. 2010. Human social evolution: a comparison of hunter-gatherer and chimpanzee social organization. Proceedings of the British Academy 158:83-113.

Le Gac, M., and M. Doebeli. 2010. Environmental viscosity does not affect the evolution of cooperation during experimental evolution of colicigenic bacteria. Evolution 64:522-533.

Lehmann, L., and L. Keller. 2006. The evolution of cooperation and altruisma general framework and a classification of models. Journal of Evolutionary Biology 19:135-176.

$\rightarrow$ Lehmann, L., and N. Perrin. 2002. Altruism, dispersal, and phenotype-matching kin recognition. American Naturalist 159:451-468.

Lehmann, L., F. Rousset, D. Roze, and L. Keller. 2007. Strong reciprocity or strong ferocity? a population genetic view of the evolution of altruistic punishment. American Naturalist 170:21-36.

Lima, F. W. S., T. Hadzibeganovic, and D. Stauffer. 2009. Evolution of ethnocentrism on undirected and directed Barabási-Albert networks. Physica A 388:4999-5004. 
play in their social networks. Proceedings of the Royal Society B: Biological Sciences 271:S477-S481.

$\rightarrow$ Mampe, B., A. G. Friederici, A. Christophe, and K. Wermke. 2009. Newborns cry melody is shaped by their native language. Current Biology 19:19941997.

Markham, D. 1999. Listeners and disguised voices: the imitation and perception of dialectal accent. International Journal of Speech Language and th Law 6:289-299.

$\rightarrow$ Marwick, B. 2003. Pleistocene exchange networks as evidence for the evolutior of language. Cambridge Archaeological Journal 13:67-81.

2005. The interpersonal origins of language: social and linguisti implications of an archaeology approach to language evolution. Language and the Human Sciences 1:197-224.

$\rightarrow$ Masuda, N., and H. Ohtsuki. 2007. Tag-based indirect reciprocity by incom plete social information. Proceedings of the Royal Society B: Biological Sciences 274:689-695.

Mauss, Marcel. 1979. The notion of body techniques. In Sociology and psychology: essays. Ben Brewster, trans. Pp. 97-123. London: Routledge \& Kegan Paul. [DD/MD]

$\rightarrow$ McBrearty, S., and A. S. Brooks. 2000. The revolution that wasn't: a new interpretation of the origin of modern human behavior. Journal of Humai Evolution 39:453-563.

$\rightarrow$ McElreath, R., R. Boyd, and P. J. Richerson. 2003. Shared norms and th evolution of ethnic markers. Current Anthropology 44:122-129.

$\rightarrow$ McNamara, T. 2005. 21st century shibboleth: language tests, identity and intergroup conflict. Language Policy 4:351-370.

$\rightarrow$ McPherson, M., L. Smith-Lovin, and J. M. Cook. 2001. Birds of a feather: homophily in social networks. Annual Review of Sociology 27:415-444.

$\rightarrow$ Mehler, J., P. Jusczyk, and G. Lambertz. 1988. A precursor of language acquisition in young infants. Cognition 29:143-178.

$\rightarrow$ Mellars, P. 2005. The impossible coincidence: a single-species model for the origins of modern human behavior in Europe. Evolutionary Anthropology 14:12-27.

$\rightarrow$ Meltzoff, A., P. Kuhl, J. Movellan, and T. J. Sejnowski. 2009. Foundations fo a new science of learning. Science 325:284-288.

Milroy, L., and P. McClenaghan. 1977. Stereotyped reactions to four educater accents in Ulster. Belfast Working Papers in Language and Linguistics 2:111.

$\rightarrow$ Milton, K. 1999. A hypothesis to explain the role of meat-eating in human evolution. Evolutionary Anthropology 8(1):11-21.

$\rightarrow$ Moen, I. 2000. Foreign accent syndrome: a review of contemporary explanations. Aphasiology 14:5-15.

$\rightarrow$ Nazzi, T., C. Floccia, and J. Bertoncini. 1998. Discrimination of pitch contours by neonates. Infant Behavior and Development 21:779-784.

$\rightarrow$ Nazzi, T., P. W. Jusczyk, and E. K. Johnson. 2000. Language discrimination by English-learning 5-month-olds: effects of rhythm and familiarity. Journal of Memory and Language 43:1-19.

$\rightarrow$ Nerbonne, J. 2010. Measuring the diffusion of linguistic change. Philosophical Transactions of the Royal Society B: Biological Sciences 365(1559):3821-3828. [QDA]

$\rightarrow$ Nettle, D. 1996. Language diversity in West Africa: an ecological approach. Journal of Anthropological Archaeology 15:403-438.

- 1999a. Language variation and the evolution of societies. In The evolution of culture. R. Dunbar, C. Knight, and C. Power, eds. Pp. 214-227. Edinburgh: Edinburgh University Press.

$\rightarrow$. 1999b. Using social impact theory to simulate language change. Lin-

gua 108:95-117.
$\rightarrow$ Nettle, D., and R. Dunbar. 1997. Social markers and the evolution of reciproca. exchange. Current Anthropology 38:93-99.

Neuhauser, S., and A. P. Simpson. 2007. Imitated or authentic? judgement. of foreign accents. International Congress of Phonetic Sciences 16:1805-1808.

$\rightarrow$ Nikolov, M., and J. M. Djigunovic. 2006. Recent research on age, seconc. language acquisition, and early foreign language learning. Annual Review of Applied Linguistics 26:234-260. $\rightarrow$ Nowak, M. A., C. E. Tarnita, and T. Antal. 2010. Evolutionary dynamics i1-
structured populations. Philosophical Transactions of the Royal Society B 365: 19-30.

Pagel, M. 2012. Wired for culture: origins of the human social mind. New York: Norton. [QDA]

$\rightarrow$ Palmer, C. T., B. E. Fredrickson, and C. T. Tilley. 1997. Categories and gatherings: group selection and the mythology of cultural anthropology. Evolution and Human Behavior 18:291-308.

$\rightarrow$ Pepper, J. W., and B. B. Smuts. 2002. A mechanism for the evolution of altruism among nonkin: positive assortment through environmental feedback. American Naturalist 160:205-213.

Powell, A., S. Shennan, and M. G. Thomas. 2009. Late Pleistocene demography and the appearance of modern human behavior. Science 324:1298-1301.

Richerson, P. J., and R. Boyd. 2005. Not by genes alone. Chicago: University of Chicago Press.

Riolo, R. L., M. D. Cohen, and R. Axelrod. 2001. Evolution of cooperation without reciprocity. Nature 414:441-443.

$\rightarrow$ Roberts, G. 2008. Language and the free-rider problem: an experimental paradigm. Biological Theory 3:174-183.

Ryan, E. B. 2008. Social psychological mechanisms underlying native speaker evaluations of non-native speech. Studies in Second Language Acquisition 5: $148-159$.

Sangrigoli, S., and S. de Schonen. 2004. Recognition of own-race and otherrace faces by three-month-old infants. Journal of Child Psychology and Psychiatry 45:1219-1227.

Sankoff, G. 1980. The social life of language. Philadelphia: University of Pennsylvania Press.

$\rightarrow$ Santos, F. C., J. M. Pacheco, and B. Skyrms. 2011. Co-evolution of pre-play signaling and cooperation. Journal of Theoretical Biology 274:30-35.

Scovel, T. 2000. A critical review of the critical period research. Annual Review of Applied Linguistics 20:213-223.

Shennan, S. 2001. Demography and cultural innovation: a model and its implications for the emergence of modern human culture. Cambridge Archaeological Journal 11:5-16.

Shultz, T. R., M. Hartshorn, and R. A. Hammond. 2008. Stages in the evolution of ethnocentrism. In Proceedings of the 30th annual conference of the Cognitive Science Society. B. C. Love, K. McRae, and V. M. Sloutsky, eds. Pp. 1244-1249. Austin, TX: Cognitive Science Society.

Shultz, T. R., M. Hartshorn, and A. Kaznatcheev. 2009. Why is ethnocentrism more common than humanitarianism? In Proceedings of the 31st annual conference of the Cognitive Science Society. N. A. Taatgen and H. van Rijn, eds. Pp. 2100-2105. Austin, TX: Cognitive Science Society.

Sigmund, K. 2009. Sympathy and similarity: the evolutionary dynamics of cooperation. Proceedings of the National Academy of Sciences 106:8405-8406.

Sigmund, K., and M. A. Nowak. 2001. Tides of tolerance. Nature 414:403404.

Silk, J., and R. Boyd. 2010. From grooming to giving blood: the origins of human altruism. In Mind the gap: tracing the origins of human universals. P. M. Kappeler and J. B. Silk, eds. Pp. 223-224. Heidelberg: Springer.

Singleton, D. 2002. Age and second language acquisition. Annual Review of Applied Linguistics 2:77-89.

Sjöström, M., E. J. Eriksson, E. Zetterholm, and K. P. H. Sullivan. 2006. A switch of dialect as disguise. In Proceedings of Fonetik 2006. Gilbert Ambrazaitis and Susanne Schötz, eds. Pp. 113-116. Lund: Lund University.

Smith, E. 2003. Human cooperation: perspectives from behavioral ecology. In Genetic and cultural evolution of cooperation. P. Hammerstein, ed. Pp. 401-427. Cambridge, MA: MIT Press.

Smith, Ian, and Steve Johnson. 1986. Sociolinguistic patterns in an unstratified society: the patrilects of Kugu Nganhcara. Journal of the Atlantic Provinces Linguistic Association 8:29-43. [DRL, LH-L]

Sober, E., and D. S. Wilson. 1998. Unto others: the evolution and psychology of unselfish behavior. Cambridge, MA: Harvard University Press.

Sosis, R., H. C. Kress, and J. S. Boster. 2007. Scars for war: evaluating alternative signaling explanations for cross-cultural variance in ritual scars. Evolution and Human Behavior 28:234-247.

Spector, L., and J. Klein. 2006. Genetic stability and territorial structure facilitate the evolution of tag-mediated altruism. Artificial Life 12:553-560.

Sperber, D., and L. A. Hirschfeld. 2004. The cognitive foundations of cultural stability and diversity. Trends in Cognitive Sciences 8:40-46.

Tagliamonte, A. S., and S. Molfenter. 2007. How'd you get that accent? acquiring a second dialect of the same language. Language in Society 36:649675.

Tajfel, H. 1982. Social psychology of intergroup relations. Annual Review of Psychology 33:1-39.

Tarnita, C. E., T. Antal, H. Ohtsuki, and M. A. Nowak. 2009. Evolutionary dynamics in set structured populations. Proceedings of the National Academy of Sciences of the USA 106:8601-8604. [AT, JG]

Tomasello, M. 1999. The cultural origins of human cognition. Cambridge, MA: Harvard University Press. Press. 2008 . The origins of human communication. Cambridge, MA: MIT 
$\rightarrow$ Tooby, J., and L. Cosmides. 1989. Kin selection, genic selection, and information-dependent strategies. Behavioral and Brain Sciences 12:542-544.

$\rightarrow$ Traulsen, A. 2008. Mechanisms for similarity based cooperation. European Physical Journal B 63:363-371.

$\rightarrow$ Traulsen, A., and J. C. Claussen. 2004. Similarity-based cooperation and spatial segregation. Physical Review E 70(4):046128.

$\rightarrow$ Traulsen, A., and M. A. Nowak. 2007. Chromodynamics of cooperation in finite populations. PLoS ONE 2:e270.

$\rightarrow$ Traulsen, A., and H. G. Schuster. 2003. Minimal model for tag-based co operation. Physical Review E 68:046129.

Trudgill, P. 2000. Sociolinguistics: an introduction to language and society. 4th ed. London: Penguin.

2011. Sociolinguistic typology: social determinants of linguistic com plexity. Oxford: Oxford University Press.

$\rightarrow$ Vanhaeren, M., and F. d'Errico. 2006. Aurignacian ethno-linguistic geography of Europe revealed by personal ornaments. Journal of Archaeological Scienc 33:1105-1128.

$\rightarrow$ West, S. A., C. El Mouden, and A. Gardner. 2010. Sixteen common misconceptions about the evolution of cooperation in humans. Evolution an Human Behavior 32:231-262.

$\rightarrow$ West, S. A., A. Gardner, D. M. Shuker, T. Reynolds, M. Burton-Chellow,
E. M. Sykes, M. A. Guinnee, and A. S. Griffin. 2006a. Cooperation and the scale of competition in humans. Current Biology 16:1103-1106.

West S. A., A. S. Griffin, and A. Gardner. 2006b. Social semantics: altruism, cooperation, mutualism, strong reciprocity and group selection. Journal of Evolutionary Biology 20:425-432.

$\rightarrow$ Wey, T. W., and D. T. Blumstein. 2010. Social cohesion in yellow-bellied marmots is established through age and kin structuring. Animal Behaviour 79:1343-1352.

$\rightarrow$ Whallon, R. 2006. Social networks and information: non-"utilitarian” mobility among hunter-gatherers. Journal of Anthropological Archaeology 25:259-270.

Wiessner, P. W. 1977. Hxaro: a regional system of reciprocity for reducing risk among the !kung San. PhD dissertation, University of Michigan, Ann Arbor.

$\rightarrow$ Wright, T. F., and G. S. Wilkinson. 2001. Population genetic structure and vocal dialects in an Amazon parrot. Proceedings of the Royal Society B 268: 609-616.

Youssouf, Ibrahim Ag, Allen D. Grimshaw, and Charles S. Bird. 1976. Greetings in the desert. American Ethnologist 3(4):797-824, doi:10.1525/ae.1976.3.4 .02a00140. [DD, MD]

Yurk, H., L. Barrett-Lennard, J. K. B. Ford, and C. O. Matkin. 2002. Cultural transmission within maternal lineages: vocal clans in resident killer whales in southern Alaska. Animal Behaviour 63:1103-1119. 